\title{
SEMI-ALGEBRAIC CHAINS ON PROJECTIVE VARIETIES AND THE ABEL-JACOBI MAP FOR HIGHER CHOW CYCLES
}

\author{
KENICHIRO KIMURA
}

\section{INTRODUCTION}

The main object of study in this paper is the cohomology groups of smooth quasiprojective complex varieties. The reader may be skeptical about finding anything new in general about this subject. What we are going to do is to describe the cohomology groups of a smooth quasi-projective variety $U$ relative to a normal crossing divisor $\mathbf{H}$, in terms of $\delta$-admissible chains. Roughly speaking, a $\delta$-admissible chain on $U$ relative to a subvariety $Y$ of $U$ is a simplicial semi-algebraic chain $\gamma$ such that the support of $\gamma$ and that of $\delta \gamma$ (the boundary of $\gamma$ ) meet $Y$ properly. One of the merits of $\delta$-admissible chains is that they admit pull-back to $Y$. By this property, for a normal crossing divisor $\mathbf{H}$ on $U$ we construct a certain complex $A C^{*}(U, \mathbf{H})$ of $\delta$-admissible chains such that we have an isomorphism

$$
H^{j}\left(A C^{*}(U, \mathbf{H})\right) \simeq H^{j}(U, \mathbf{H} ; \mathbb{Z}) .
$$

The proof is not quite elementary, and we needed to use some sheaf theory.

We can describe the duality pairing between the de Rham and the singular cohomology via integral on $\delta$-admissible chains. Let $\varphi$ be a smooth $p$-form on $U$ with compact support which has logarithmic singularity along $\mathbf{H}$, and let $\gamma$ be a $\delta$-admissible $(p+1)$-chain on $U$. Then in Proposition 3.2 we show that the equality

$$
\int_{\partial_{\mathbf{H} \gamma}} \operatorname{Res} \varphi=\int_{\delta \gamma} \varphi-\int_{\gamma} d \varphi
$$

holds. For the definition of the differential $\partial_{\mathbf{H}}$ see Definition 2.16. This formula is a generalization of the Stokes formula, and we call this equality the Cauchy-Stokes formula. Note that the integral of a differential form with $\log$ poles on a $C^{\infty}$ - chain does not necessarily converge, even if the chain meets the faces properly. See the example in Remark 3.3 (1). By the Cauchy-Stokes formula we have a certain pairing

$$
A C^{*}(U, \mathbf{H}) \otimes A_{c}^{*}(U)(\log \mathbf{H}) \rightarrow \mathbb{C}[-2 \operatorname{dim} U]
$$

which is a map of complexes, and this induces the duality pairing

$$
H^{2 \operatorname{dim} U-j}(U, \mathbf{H} ; \mathbb{Q}) \otimes H^{j}(X-\mathbf{H}, D ; \mathbb{C}) \rightarrow \mathbb{C} .
$$

Here $X$ is a smooth projective complex variety, $D$ is a closed subset of $X$ such that $X-D$ is isomorphic to $U$. As an application, in $\S 4$ we show that the Abel-Jacobi map for higher Chow cycles can be described in terms of $\delta$-admissible chains, which can be regarded as a natural generalization of the original definition by Griffiths of the Abel-Jacobi map for ordinary algebraic cycles. Details are in $\S 4$, but we point out one advantage of the admissible chains. Let $Y$ be a smooth projective complex variety, and $Z \in z^{p}(Y, n)^{\text {alt }}$ be a higher 
Chow cycle such that $\partial_{\square} Z=0$. See $\S 4$ for the definitions. As is described in [2] and [16, the definition of the Abel-Jacobi map starts with defining a cohomology class of $Z$ in $H_{|Z|}^{2 p}\left(\square^{n} \times Y, \partial \square^{n} \times Y ; \mathbb{Q}(p)\right)$, which is the cohomology of $\square^{n} \times Y$ with support on $Z$, relative to the faces of $\square^{n} \times Y$. By using the admissible chains we can construct a certain complex which computes this cohomology group, such that the class of the cycle $Z$ is represented by $Z$ itself. If $Z$ is homologous to zero, then there is a chain $\Gamma$ which has $Z$ as the boundary. It is an immediate consequence of the construction that the class of $\Gamma$ gives the Abel-Jacobi image of $Z$. This construction works also for open varieties, and for relative higher Chow cycles. As an example, we will see that the Hodge realization of the cycles of polylogarithms constructed in [3] can be described in terms of the Abel-Jacobi map of certain open varieties.

The problem of describing the Abel-Jacobi maps for higher Chow cycles has been considered by other authors too. This paper is partly inspired by [10] and [11]. Our description of the Abel-Jacobi map can be regarded as a generalization of the geometric interpretation in $\S 5.8$ of [10] to quasi-projective varieties. Even in the case of projective varieties, our description is somewhat different from the one given in [10].

\section{The COMPleX of ADMissible Chains}

Let $k$ be a non-negative integer. A $k$-simplex in an Euclidean space $\mathbb{R}^{n}$ is the convex hull of affinely independent points $a_{0}, \cdots, a_{k}$ in $\mathbb{R}^{n}$. A finite simplicial complex of $\mathbb{R}^{n}$ is a finite set $K$ consisting of simplexes such that (1) for all $s \in K$, all the faces of $s$ belong to $K,(2)$ for all $s, t \in K, s \cap t$ is either the empty set or a common face of $s$ and $t$. We denote by $K_{p}$ the set of $p$-simplexes of $K$. For a finite simplicial complex $K$, the union of simplexes in $K$ as a subset of $\mathbb{R}^{n}$ is denoted by $|K|$.

As for the definition of semi-algebraic set and their fundamental properties, see [4].

Theorem 2.1 ([4], Theorem 9.2.1). Let $P$ be a compact semi-algebraic subset of $\mathbb{R}^{m}$. The set $P$ is triangulable, i.e. there exists a finite simplicial complex $K$ and a semi-algebraic homeomorphism $\Phi_{K}:|K| \rightarrow P$. Moreover, for a given finite family $S=\left\{S_{j}\right\}_{j=1, \cdots, q}$ of semi-algebraic subsets of $P$, we can choose a finite simplicial complex $K$ and a semi-algebraic homeomorphism $\Phi_{K}:|K| \rightarrow P$ such that every $S_{j}$ is the union of a subset of $\left\{\Phi_{K}\left(\sigma^{\circ}\right)\right\}_{\sigma \in K}$. Here $\sigma^{\circ}$ is the interior of $\sigma$, which is the complement of the union of all proper faces of $\sigma$.

Remark 2.2. (1) By [4] Remark 9.2.3 (a), the map $\Phi_{K}$ can be taken so that the map $\Phi_{K}$ is facewise regular embedding i.e. for each $\sigma \in K, \Phi_{K}\left(\sigma^{\circ}\right)$ is a regular submanifold of $\mathbb{R}^{m}$.

(2) The pair $\left(K, \Phi_{K}\right)$ as in Theorem 2.1 is called a semi-algebraic triangulation of $P$; we will then identify $|K|$ with $P$. A projective real or complex variety $V$ is regarded as a compact semi-algebraic subset of an Euclidean space by [4] Theorem 3.4.4, thus the above theorem applies to $V$.

We recall some terminology of piecewise-linear topology. Let $K$ be a simplicial complex and $L$ be a subcomplex of $K$. L is a full subcomplex of $K$, if all the vertices of a simplex $\sigma$ in $K$ belong to $L$, then $\sigma$ belongs to $L$. a derived subdivision of $K \bmod L$ is obtained by starring each simplex of $K$ not contained in $L$. See for example [15] page 20 for more detail. If we star each simplex not contained in $L$ at its barycenter, we obtain the barycentric subdivision of $K \bmod L$, which is denoted by $\operatorname{sd} K \bmod L$. The simplicial neighborhood of $L$ in $K$, denoted by $N(L, K)$, is defined to be $\{\sigma \in K \mid \exists \eta, \sigma$ is a face of $\eta$ and $\eta \cap L \neq \emptyset\}$. Also the simplicial 
complement of $L$ in $K$, denoted by $C(L, K)$, is defined to be $\{\sigma \in K \mid \sigma \cap L=\emptyset\}$. The intersection $N(L, K) \cap C(L, K)$ is denoted by $\dot{N}(L, K)$. Suppose that $L$ is a full subcomplex of $K$, and let $K^{\prime}$ be a derived subdivision of $K \bmod L \cup C(L, K)$. The polytope $\left|N\left(L, K^{\prime}\right)\right|$ is said to be a regular neighborhood of $|L|$ in $|K|$.

Notation 2.3. Let $K$ be a simplicial complex. For a subcomplex $L$ of $K$, the space $|L|$ is a subspace of $|K|$. A subset of $|K|$ of the form $|L|$ is also called a subcomplex. If a subset $S$ of $|K|$ is equal to $|M|$ for a subcomplex $M$ of $K$, then $M$ is often denoted by $K \cap S$. For two simplexes $\sigma$ and $\eta$ we denote $\sigma \prec \eta$ if $\sigma$ is a face of $\eta$. For a complex $K$ and its subcomplex $L$, by writing $L \triangleleft K$ we mean that $L$ is a full subcomplex of $K$.

Let $X$ be a smooth projective variety over $\mathbb{C}$ of dimension $m, D$ be a closed subset of $X$ and $\mathbf{H}=H_{0} \cup \cdots \cup H_{t}$ be a strict normal crossing divisor on $X$. We write $U=X-D$. The faces of $X$ are intersections of several $H_{j}$ 's. For a subset $I$ of $T:=\{0, \cdots, t\}$, we write the face $\cap_{i \in I} H_{i}$ by $H_{I}$. In the following we suppose that each triangulation $K$ of $X$ is semi-algebraic, and that the subset $D$ is a subcomplex of $K$. Let $K$ be a triangulation of $X$. We denote by $C_{\bullet}(K ; \mathbb{Z})$ resp. $C_{\bullet}(K, D ; \mathbb{Z})$ the chain complex resp. the relative chain complex of $K$. An element of $C_{p}(K ; \mathbb{Z})$ for $p \geq 0$ is written as $\sum a_{\sigma} \sigma$ where the sum is taken over $p$-simplexes of $K$. By doing so, it is agreed upon that an orientation has been chosen for each $\sigma$. By abuse of notation, an element of $C_{\bullet}(K, D ; \mathbb{Z})$ is often described similarly.

Definition 2.4. For an element $\gamma=\sum a_{\sigma} \sigma$ of $C_{p}(K ; \mathbb{Z})$, we define the support $|\gamma|$ of $\gamma$ as the subset of $|K|$ given by

$$
|\gamma|=\bigcup_{\substack{\sigma \in K_{p} \\ a_{\sigma} \neq 0}} \sigma .
$$

For an element $\gamma=\sum a_{\sigma} \sigma$ of $C_{p}(K ; \mathbb{Z}),|\gamma|$ is sometimes regarded as a subcomplex of $K$.

Definition 2.5. Let $p \geq 0$ be an integer.

(1) (Admissibility) A semi-algebraic subset $S$ of $X$ is said to be admissible if for each face $H$, the inequality

$$
\operatorname{dim}(S \cap(H-D)) \leq \operatorname{dim} S-2 \operatorname{codim} H
$$

holds. Here note that $\operatorname{dim}(S \cap(H-D))$ and $\operatorname{dim} S$ are the dimensions as semialgebraic sets, and codim $H$ means the codimension of the subvariety $H$ of $X$.

(2) Let $\gamma$ be an element of $C_{p}(K, D ; \mathbb{Z})$. Then $\gamma$ is said to be admissible if the support of a representative of $\gamma$ in $C_{p}(K ; \mathbb{Z})$ is admissible. This condition is independent of the choice of a representative.

(3) We set

$$
\left.A C_{p}(K, D ; \mathbb{Z})=\left\{\gamma \in C_{p}(K, D ; \mathbb{Z})\right) \mid \gamma \text { and } \delta \gamma \text { are admissible }\right\} .
$$

We call an element of $A C_{p}(K, D ; \mathbb{Z})$ a $\delta$-admissible chain.

\subsection{Subdivision and inductive limit.}

Definition 2.6. Let $\left(K, \Phi_{K}:|K| \rightarrow P\right)$ be a triangulation of a compact semi-algebraic set $P$. Another triangulation $\left(K^{\prime}, \Phi_{K^{\prime}}:\left|K^{\prime}\right| \rightarrow P\right)$ is a subdivision of $K$ if : 
(1) The image of each simplex of $K^{\prime}$ under the map $\Phi_{K^{\prime}}$ is contained in the image of a simplex of $K$ under the map $\Phi_{K}$.

(2) The image of each simplex of $K$ under the map $\Phi_{K}$ is the union of the images of simplexes of $K^{\prime}$ under $\Phi_{K^{\prime}}$.

If $K^{\prime}$ is a subdivision of a triangulation $K$, there is a natural homomorphism of complexes $\lambda: C_{\bullet}(K, D ; \mathbb{Z}) \rightarrow C_{\bullet}\left(K^{\prime}, D ; \mathbb{Z}\right)$ called the subdivision operator. See for example 14] Theorem 17.3 for the definition. For a simplex $\sigma$ of $K$, the chain $\lambda(\sigma)$ is carried by $K^{\prime} \cap \sigma$, and so that the map $\lambda$ sends $A C \bullet(K, D ; \mathbb{Z})$ to $A C \bullet\left(K^{\prime}, D ; \mathbb{Z}\right)$. By Theorem 2.1 two semialgebraic triangulations have a common subdivision. Since the map $\lambda$ and the differential $\delta$ commute, the complexes $C_{\bullet}(K, D ; \mathbb{Z})$ and $A C_{\bullet}(K, D ; \mathbb{Z})$ form inductive systems indexed by triangulations $K$ of $X$.

Definition 2.7. We set

$$
C_{\bullet}(X, D ; \mathbb{Z})=\underset{\mathrm{K}}{\lim } C_{\bullet}(K, D ; \mathbb{Z}), \quad A C \bullet(X, D ; \mathbb{Z})=\underset{\mathrm{K}}{\lim } A C \bullet(K, D ; \mathbb{Z}) .
$$

Here the limit is taken on the directed set of triangulations.

A proof of the following Proposition is given in [8] Appendix A.

Proposition 2.8 (Moving lemma). The inclusion of complexes

$$
\iota: A C_{\bullet}(X, D ; \mathbb{Z}) \rightarrow C_{\bullet}(X, D ; \mathbb{Z})
$$

is a quasi-isomorphism.

Definition 2.9 (Good triangulation). We define a family $\mathcal{L}$ of subsets of $X$ by

$$
\mathcal{L}=\left\{H_{I_{1}} \cup \cdots \cup H_{I_{k}}\right\}_{\left(I_{1}, \ldots, I_{k}\right)},
$$

where $H_{I_{j}}$ are faces of $X$. In short, a member of $\mathcal{L}$ is the union of several faces. A finite semi-algebraic triangulation $K$ of $X$ is called a good triangulation if $K$ satisfies the following conditions.

(1) The divisor $D$ is a subcomplex of $K$.

(2) The map $\Phi_{K}:|K| \rightarrow X$ is facewise regular embedding. cf. Remark 2.2.

(3) Each element $L_{i} \in \mathcal{L}$ is a full subcomplex of $K$ i.e. there exists a full subcomplex $M_{i}$ of $K$ such that $L_{i}=\left|M_{i}\right|$.

In particular, if $K$ is a good triangulation, then for any simplex $\sigma$ of $K$ and $L_{i} \in \mathcal{L}$, the intersection $\sigma \cap L_{i}$ is a (simplicial) face of $\sigma$. This is the primary reason to consider the condition (3).

Remark 2.10. If $L$ is a subcomplex of $K$, then $\operatorname{sd} L$ is a full subcomplex of $\operatorname{sd} K$ (See for example Exercise 3.2 of [15]). It follows that if $K$ is a semi-algebraic triangulation of $X$ which is a facewise regular embedding, and such that $D$ and each $L_{i} \in \mathcal{L}$ are subcomplexes of $K$, then $\operatorname{sd} K$ is a good triangulation.

In the following, each triangulation $K$ of $X$ is assumed to be good in the sense of Definition 2.9.

\subsection{The cap product with a Thom cocycle.}




\subsubsection{Simplicial cap product.}

Definition 2.11 (Ordering of complex, good ordering). Let $K$ be a good triangulation of $X$.

(1) A partial ordering on the set of vertices in $K$ is called an ordering of $K$, if the restriction of the ordering to each simplex is a total ordering.

(2) Let $L$ be a subcomplex of $K$. An ordering of $K$ is said to be good with respect to $L$ if it satisfies the following condition. If a vertex $v$ is on $L$ and $w \geq v$ for a vertex $w$, then $w \in L$.

We denote by $\left[a_{0}, \cdots, a_{k}\right]$ the simplex spanned by $a_{0}, \cdots, a_{k}$. Let $\mathcal{O}$ be a good ordering of $K$ with respect to a subcomplex $L$. We recall the definition of the cap product

$\stackrel{\mathcal{O}}{\cap}: C^{p}(K) \otimes C_{k}(K) \rightarrow C_{k-p}(K)$. For a simplex $\alpha=\left[v_{0}, \cdots, v_{k}\right]$ such that $v_{0}<\cdots<v_{k}$ and $u \in C^{p}(K)$, we define

$$
u \stackrel{\mathcal{O}}{\cap} \alpha=u\left(\left[v_{0}, \ldots, v_{p}\right]\right)\left[v_{p}, \ldots, v_{k}\right] .
$$

One has the boundary formula

$$
\delta(u \stackrel{\mathcal{O}}{\cap} \alpha)=(-1)^{p}(u \stackrel{\mathcal{O}}{\cap}(\delta \alpha)-(d u) \stackrel{\mathcal{O}}{\cap} \alpha)
$$

where $d u$ denotes the coboundary of $u$, see [9], p.239 (note the difference in sign convention from [14]). Thus if $u$ is a cocycle, $\delta(u \stackrel{\mathcal{O}}{\cap} \alpha)=(-1)^{p} u \stackrel{\mathcal{O}}{\cap}(\delta \alpha)$.

2.2.2. Cohomology class of a subvariety. For a subvariety $V$ of $X$ of codimension $p$, there exists a cohomology class $c l(V) \in H_{V}^{2 p}(X, \mathbb{Z})$. In the case of simplicial cohomology, it is described as follows. Let $K$ be a triangulation of $X$ such that there exists a full subcomplex $L$ of $K$ with $V=|L|$. Let $K^{\prime}$ be a derived subdivision of $K \bmod L \cup C(L, K)$. Set $N=N\left(L, K^{\prime}\right)$ and $C=C\left(L, K^{\prime}\right)$. In this situation the cohomology group $H_{V}^{2 p}(X, \mathbb{Z})$ is equal to $H^{2 p}\left(C^{*}(K, C ; \mathbb{Z})\right)$, and by Lefschetz duality Theorem 3.43 [9] we have an isomorphism

$$
\cap \eta_{X}: H^{2 p}\left(C^{*}(K, C ; \mathbb{Z})\right) \rightarrow H_{2(m-p)}\left(C_{*}(N ; \mathbb{Z})\right) .
$$

Here $\eta_{X}$ is the fundamental cycle of $X$. The element $\operatorname{cl}(V)$ is the one such that $c l(V) \cap \eta_{X}$ equals the homology class of the cycle $V$. A cocycle in $C^{2 p}(K, C ; \mathbb{Z})$ which represents $c l(V)$ is called a Thom cocycle of $V$, and is denoted by $T_{V}$.

Proposition 2.12. Let $V$ be a subvariety of $X$ of codimension $p, K$ a triangulation of $X$ for which $V=|L|$ for a full subcomplex $L$. Let $K^{\prime}$ be a derived subdivision of $K$ mod $L \cup C(L, K), T_{V} \in C^{2 p}\left(K, C\left(L, K^{\prime}\right) ; \mathbb{Z}\right)$ be a Thom cocycle of $V$ and $\mathcal{O}$ a good ordering of $K^{\prime}$ with respect to $L$.

(1) The map $T_{V} \stackrel{\mathcal{O}}{\cap}$ and the topological differential $\delta$ commute.

(2) The image of the homomorphism $T_{V} \stackrel{\mathcal{O}}{\cap}$ is contained in $C_{k-2 p}(L ; \mathbb{Z})$, As a consequence, we have a homomorphism of complexes

$$
T_{V} \stackrel{\mathcal{O}}{\cap}: C_{k}\left(K^{\prime}, D ; \mathbb{Z}\right) \rightarrow C_{k-2 p}(L, L \cap D ; \mathbb{Z}) .
$$


Proof. (1). Since $T_{V}$ is a cocycle of even degree, we have $\delta\left(T_{V} \stackrel{\mathcal{O}}{\cap} \sigma\right)=T_{V} \stackrel{\mathcal{O}}{\cap}(\delta \sigma)$ for a simplex $\sigma$.

(2). Let $\sigma=\left[v_{0}, \cdots, v_{n}\right] \in C_{n}\left(K^{\prime}\right)$ with $v_{0}<\cdots<v_{n}$. If $v_{2 p} \notin V$, then $\left[v_{0}, \cdots, v_{2 p}\right] \cap V=\emptyset$ and $T_{V}\left(\left[v_{0}, \cdots, v_{2 p}\right]\right)=0$ since the cochain $T$ vanishes on $C\left(L, K^{\prime}\right)$. If $v_{2 p} \in L$, then the vertices $v_{2 p}, \cdots, v_{n}$ are on $L$, and we have $\left[v_{2 p}, \ldots, v_{n}\right] \subset L$ since $L \triangleleft K$. Thus the assertion holds.

2.2.3. Independence of $T_{V}$ and ordering.

Proposition 2.13. Let $K$ be a good triangulation of $X$ and $H$ be a codimension $p$ face of $X$. Set $L=K \cap H$. Let $T_{H} \in C^{2 p}\left(K^{\prime}, C\left(L, K^{\prime}\right) ; \mathbb{Z}\right)$ be a Thom cocycle of $H$, O a good ordering of $K^{\prime}$ with respect to $L$, and $\gamma$ be an element of $A C_{k}\left(K^{\prime}, D ; \mathbb{Z}\right)$. Then we have the following.

(1) The chain $T_{H} \stackrel{\mathcal{O}}{\cap} \gamma$ is an element in $A C_{k-2 p}(L, L \cap D ; \mathbb{Z})$.

(2) The chain $T_{H} \stackrel{\mathcal{O}}{\cap} \gamma$ is independent of the choice of a Thom cocycle $T_{H}$ and a good ordering $\mathcal{O}$. Thus the map

$$
T_{H} \stackrel{\mathcal{O}}{\cap}: A C_{k}(K, D ; \mathbb{Z}) \rightarrow A C_{k-2 p}(L, L \cap D ; \mathbb{Z}) .
$$

induced by (2.2.5) is denoted by $T_{H} \cap$.

(3) Let $M$ be a good subdivision of $K$. Let $M^{\prime}$ be a derived subdivision of $M$ mod $(M \cap H) \cup C(M \cap H, M)$ and $T_{H}^{\prime} \in C^{(2 p)}\left(M^{\prime}, C\left(M \cap H, M^{\prime}\right) ; \mathbb{Z}\right)$ be a Thom cocycle of $H$. Then we have the following commutative diagram.

$$
\begin{array}{ccc}
A C_{k}(K, D ; \mathbb{Z}) & \stackrel{T_{H} \cap}{\longrightarrow} & A C_{k-2 p}(L, L \cap D ; \mathbb{Z}) \\
\lambda \downarrow & & \downarrow \lambda \\
A C_{k}\left(M^{\prime}, D ; \mathbb{Z}\right) & \stackrel{T_{H}^{\prime} \cap}{\longrightarrow} & A C_{k-2 p}\left(M^{\prime} \cap H, M^{\prime} \cap(D \cap H) ; \mathbb{Z}\right)
\end{array}
$$

where the vertical maps $\lambda$ are subdivision operators.

Proof. (1). For an element $z \in C_{k}\left(K^{\prime}, D ; \mathbb{Q}\right)$, we have $T_{H} \stackrel{\mathcal{O}}{\cap} z \in C_{k-2 p}(L, D ; \mathbb{Q})$ by Proposition 2.12 (2). By the definition of the cap product, we see that the set $\left|T_{H} \stackrel{\mathcal{O}}{\cap} z\right| \subset|z| \cap H$. It follows that if $z$ is admissible i.e. $|z|-D$ meets all the faces properly, then $\left|T_{H} \stackrel{\mathcal{O}}{\cap} z\right|-D$ meets all the faces of $H$ properly. Similarly, if the chain $\delta z$ is admissible, then $T_{H} \stackrel{\mathcal{O}}{\cap}(\delta z)$ is admissible in $H$. By Proposition 2.12 (1) we have the equality $\delta\left(T_{H} \stackrel{\mathcal{O}}{\cap} z\right)=T_{H} \stackrel{\mathcal{O}}{\cap}(\delta z)$.

(2) A proof is given in [8] Section B.2.

(3) A proof is given in [8] Section B.3.

By taking the inductive limit of the homomorphism

$$
T_{H} \cap: A C_{\bullet}(K, D ; \mathbb{Q}) \rightarrow A C \bullet-2 p(L, D \cap L ; \mathbb{Q}) .
$$

for subdivisions, we get a homomorphism

$$
T_{H} \cap: A C_{\bullet}(X, D ; \mathbb{Q}) \rightarrow A C_{\bullet-2 p}(H, D \cap H ; \mathbb{Q}) .
$$


Definition 2.14. The map $T_{H} \cap$ of (2.2.7) is denoted by $\partial_{H}$, and called the face map of the face $H$.

Proposition 2.15. Let $H_{1}$ resp. $H_{2}$ be a face of codimension $p_{1}$ resp. $p_{2}$ which meet properly with each other. Set $H_{12}=H_{1} \cap H_{2}$. We have the equality

$$
\partial_{H_{1}} \partial_{H_{2}}=\partial_{H_{2}} \partial_{H_{1}}, A C_{j}(K, D ; \mathbb{Q}) \rightarrow A C_{j-2\left(p_{1}+p_{2}\right)}\left(K \cap H_{12}, D \cap H_{12} ; \mathbb{Q}\right) .
$$

Proof. A proof is given in [8] Section B.4.

Definition 2.16. Let $A C^{* * *}(U)$ be the double complex defined by

$$
A C^{p, q}(U)=\underset{\sharp I=p}{\bigoplus} A C_{2 m-2 p-q}\left(H_{I}, D \cap H_{I} ; \mathbb{Z}\right)
$$

where the first differential $\partial_{\mathbf{H}}$ is defined by

$$
\left(\partial_{\mathbf{H}} \gamma\right)_{\left(\alpha_{0}<\cdots<\alpha_{p}\right)}=\sum_{i=0}^{p}(-1)^{i} \partial_{H_{\alpha_{i}}}\left(\gamma_{\left(\alpha_{0}<\cdots \widehat{\alpha_{i}} \cdots<\alpha_{p}\right)}\right)
$$

and the second differential is $\delta$ (the topological differential). For a finite set $I, \sharp I$ denotes the cardinality of $I$. The simple complex associated to $A C^{*, *}(U)$ is denoted by $A C^{*}(U, \mathbf{H} ; \mathbb{Z})$.

Theorem 2.17. For a non-negative integer $j$, we have an isomorphism

$$
H^{j}(U, \mathbf{H} ; \mathbb{Z}) \simeq H^{j}\left(A C^{*}(U, \mathbf{H} ; \mathbb{Z})\right) .
$$

Here cohomology on the left hand side is the singular cohomology of $U$ relative to $\mathbf{H}$.

Proof.

Definition 2.18. Let $V$ be an open subset of $X$. For a semi-algebraic triangulation $K$ of $X$ , we denote by $C_{*}(K,(X-V))$ resp. $A C_{*}(K,(X-V))$ the quotient of $C_{*}(K)$ resp. $A C_{*}(K)$ by the chains contained in $(X-V)$. More precisely,

$$
\begin{aligned}
& A C_{j}(K,(X-V)) \\
= & \frac{\left\{\gamma \in C_{j}(K)|| \gamma \mid-D \text { and }|\delta \gamma|-D \text { meet the faces properly }\right\}}{\{\text { those } \gamma \text { such that }|\gamma| \subset X-V\} .}
\end{aligned}
$$

The associated sheaf to the presheaf $V \mapsto \underset{K}{\lim } C_{*}(K,(X-V)) \operatorname{resp} . V \mapsto \underset{K}{\lim } A C_{*}(K,(X-V))$ is denoted by $\mathcal{C}_{*}$ resp. $\mathcal{A C}_{*}$. Here the limit is taken over semi-algebraic subdivisions of $X$. We define the associated cohomological complex by $\mathfrak{C}^{i}=\mathcal{C}_{-i}$ resp. $\mathcal{A C}^{i}=\mathcal{A C}_{-i}$.

Proposition 2.19. The complexes of sheaves $\mathcal{C}^{*}[-2 m]$ and $\mathcal{A C}^{*}[-2 m]$ are resolutions of the constant sheaf $\mathbb{Z}$.

Proof. We give a proof of the case of $\mathcal{A C}^{*}[-2 m]$. The proof for $\mathcal{C}^{*}[-2 m]$ is similar and simpler. We recall the following theorem.

Theorem 2.20. (18] Ch.6, Theorem 15) Let $M$ be a compact PL-manifold. Let $X, X_{0}$ and $H$ be subpolyhedra of $M$ such that $X_{0} \subset X$ and $X-X_{0} \subset \stackrel{\circ}{M}$ (the interior of $M$ ). Then there exists an ambient $P L$ isotopy $h: M \times[0,1] \rightarrow M$ which fixes $X_{0}$ and $\dot{M}$ (the boundary of $M)$, and such that $h_{1}\left(X-X_{0}\right)$ is in general position with respect to $H$ i.e. the inequality

$$
\operatorname{dim}\left(h_{1}\left(X-X_{0}\right) \cap H\right) \leq \operatorname{dim}\left(X-X_{0}\right)+\operatorname{dim} H-\operatorname{dim} M
$$

holds. Here $h_{t}(m)=h(m, t)$ for $m \in M$ and $t \in[0,1]$. 
The isotopy $h$ can be made arbitrarily small in the following sense. Given a positive number $\epsilon>0$, there exists a $P L$ isotopy $h$ as above such that for any point $(x, t) \in M \times[0,1]$, the inequality $\left|h_{0}(x)-h_{t}(x)\right|<\epsilon$ holds. Here $|\cdot|$ is the norm of the Euclidean space in which $M$ is contained.

For a point $x \in X$ and its neighborhood $V_{x}$, let $\gamma \in A C_{j}\left(K,\left(X-V_{x}\right)\right)$ be a cycle i.e. $|\delta \gamma| \subset\left(X-V_{x}\right)$. There exists a subdivision $K^{\prime}$ of $K$ of which $x$ is a vertex of $K^{\prime}$, and the simplicial neighborhood of $x$ in $K^{\prime}$ is contained in $V_{x}$. We replace $V_{x}$ with the interior of the simplicial neighborhood of $x$. By excision, we have

$$
H_{j}\left(X,\left(X-V_{x}\right) ; \mathbb{Z}\right) \simeq H_{j}\left(V_{x}, \delta V_{x} ; \mathbb{Z}\right)= \begin{cases}0 & j \neq 2 m \\ \mathbb{Z} & j=2 m .\end{cases}
$$

It follows that if $j \neq 2 m$, there exists $\Gamma \in C_{j+1}\left(K^{\prime}\right)$ such that $|\delta \Gamma-\gamma| \subset\left(X-V_{x}\right)$. By applying Theorem 2.20 to the case where $X=|\Gamma| \cup|\gamma| \cup D$ and $X_{0}=|\gamma| \cup D$, we can move $\Gamma$ to a chain $\Gamma^{\prime}$ keeping $|\gamma| \cup D$ fixed, so that $\left|\Gamma^{\prime}\right|-D$ and $\left|\delta \Gamma^{\prime}\right|-D$ meet the faces properly, and for a smaller neighborhood $V_{x}^{\prime}$ of $x$, we have $\left|\delta \Gamma^{\prime}-\gamma\right| \subset\left(X-V_{x}^{\prime}\right)$. For $j=2 m$, the fundamental class of $X$ gives the inclusion $\mathbb{Z} \rightarrow \mathcal{A C}_{2 m}$, the image of which is isomorphic to $H_{2 m}\left(V_{x}, \delta V_{x} ; \mathbb{Z}\right)$.

Proposition 2.21. Let $j: U \rightarrow X$ be the inclusion map. The complex of sheaves $j^{*} \mathrm{C}^{*}$ is a complex of fine sheaves.

Proof. We recall the definition of a fine sheaf from [17] page 74 Definition. A sheaf $\mathcal{F}$ on a space $X$ is fine if, for every locally finite covering $\left\{U_{\alpha}\right\}$ of $X$, there exist endomorphisms $l_{\alpha}: \mathcal{F} \rightarrow \mathcal{F}$ such that

(1) The support of $l_{\alpha}$ is contained in the closure of $U_{\alpha}$.

(2) $\sum l_{\alpha}=\mathrm{id}$.

Let $\left\{U_{\alpha}\right\}$ be a locally finite covering of $U$. Let $(K, M)$ be a semi-algebraic triangulation of the pair $(X, D)$ such that $M \triangleleft K$. Let $\pi: K \rightarrow[0,1]$ be the simplicial map defined on each vertex $v$ by

$$
\pi(v)= \begin{cases}0 & v \in M \\ 1 & v \notin M\end{cases}
$$

Since $M \triangleleft K, \pi^{-1}\{0\}=M$. By abuse of notation, the map of polytopes $X=|K| \rightarrow[0,1]$ induced by $\pi$ is also denoted by $\pi$. Inductively we will construct subdivisions $K_{n}$ ( $n=$ $1,2, \cdots)$ of $K$ with the following property.

There is a full subcomplex $L_{n}$ of $K_{n}$ such that $\left|L_{n}\right| \supset \pi^{-1}([1 / n, 1])$ and each simplex $\sigma \in L_{n}$ is contained in a $U_{\alpha}$.

Let $\operatorname{sd} K$ be the barycentric subdivision of $K$. Since the polytope $\left|\pi^{-1}\{1\}\right|=|C(M, K)|$ is compact, there exists an $m$ such that each simplex in $\operatorname{sd}^{m}\left(\pi^{-1}\{1\}\right)$ is contained in a $U_{\alpha}$. The complex $K_{1}$ is defined to be $\operatorname{sd}^{m} K$ for the smallest $m$ with the property, and $L_{1}$ is defined to be $\mathrm{sd}^{m}\left(\pi^{-1}\{1\}\right)$. Suppose that $K_{n}$ and $L_{n}$ has been constructed. We denote by $\operatorname{sd}^{m} K_{n} \bmod L_{n}$ the $m$-th barycentric subdivision of $K_{n} \bmod L_{n}$.

Lemma 2.22. For sufficiently large $m$, each simplex $\sigma \in N\left(\pi^{-1}\left[\frac{1}{n+1}, 1\right], \operatorname{sd}^{m} K_{n} \bmod L_{n}\right)$ is contained in a $U_{\alpha}$. 
Proof. For a simplex $\sigma$ of $K_{n}, \sigma \cap L_{n}$ is either empty or is a face of $\sigma$ since $L_{n} \triangleleft K_{n}$. Suppose that $\sigma \cap L_{n}=: \tau$ is not empty. If $\sigma \notin L_{n}$, then $\sigma$ is the join $\tau * \eta$ for a face $\eta$ with $\eta \cap L_{n}=\emptyset$. Let $p r: \sigma \rightarrow[0,1]$ be the simplicial map such that $p r^{-1}\{0\}=\tau$ and $p r^{-1}\{1\}=\eta$. Then we see by induction on $k$ that $N\left(\tau, \operatorname{sd}^{k} \sigma \bmod \tau\right) \subset \operatorname{pr}^{-1}\left(\left[0,\left(\frac{\operatorname{dim} \sigma}{\operatorname{dim} \sigma+1}\right)^{k}\right]\right)$. By the induction hypothesis $\tau$ is contained in a $U_{\alpha}$. Hence for a sufficiently large $m\left|N\left(\tau, \operatorname{sd}^{k} \sigma \bmod \tau\right)\right| \subset U_{\alpha}$. The set $\left|N\left(\pi^{-1}\left[\frac{1}{n+1}, 1\right], \operatorname{sd}^{m} K_{n} \bmod L_{n}\right)\right|$ is a compact subset of $U$. It follows that for a possibly larger $m$ each simplex of $N\left(\pi^{-1}\left[\frac{1}{n+1}, 1\right], \operatorname{sd}^{m} K_{n} \bmod L_{n}\right)$ is contained in a $U_{\alpha}$. By Lemma 3.3 (a) [15], There is a subdivision of $\operatorname{sd}^{m} K_{n} \bmod L_{n}$ of which $N\left(\pi^{-1}\left[\frac{1}{n+1}, 1\right], \operatorname{sd}^{m} K_{n} \bmod L_{n}\right)$ is a full subcomplex. Let $K_{n+1}$ be such a subdivision, and $L_{n+1}$ is defined to be $N\left(\pi^{-1}\left[\frac{1}{n+1}, 1\right], \operatorname{sd}^{m} K_{n} \bmod L_{n}\right)$.

Let $L_{\infty}=\cup_{n} L_{n}$. Then $L_{\infty}$ is a triangulation of $U$ each simplex of which is contained in a $U_{\alpha}$. For each $\sigma \in L_{\infty}$, choose one such $\alpha_{0}$ and define $l_{\alpha}(\sigma)=\left\{\begin{array}{ll}\sigma & \alpha=\alpha_{0} \\ 0 & \alpha \neq \alpha_{0}\end{array}\right.$. Let $x \in U$ be a point, and $s \in\left(\mathcal{C}_{j}\right)_{x}$ an element of the stalk of the sheaf $\mathcal{C}_{j}$ at $x . s$ is the restriction of an element $S \in C_{j}\left(K,\left(X-V_{x}\right)\right)$ for a neighborhood $V_{x}$ of $x$ and for a triangulation $K$ of $X$. We can assume that $V_{x} \subset\left|L_{n}\right|$ for an $n$. $S$ is a sum $\sum a_{\sigma} \sigma$. Taking $V_{x}$ smaller if necessary, we can assume that each $\sigma$ contains $x$. Take a common subdivision $K^{\prime}$ of $K$ and $K_{n}$. For each simplex $\tau$ of $K$ and $\eta$ of $K_{n}$, the intersection $\tau \cap \eta$ is the union of interior of several simplexes of $K^{\prime}$. For a simplex $\sigma \in K$ with $a_{\sigma} \neq 0$, we have $\sigma \cap L_{n}=\sum \tau_{m} \bmod X-V_{x}$ with each $\tau_{m}$ a $j$-simplex of $K^{\prime}$ since $V_{x}$ is an neighborhood of $x$ in $U$. For each $\tau_{m}$, let $\eta_{m}$ the smallest simplex of $K_{n}$ which contains $\tau_{m}$. Then we define

$$
l_{\alpha}\left(\tau_{m}\right)= \begin{cases}\tau_{m} & l_{\alpha}\left(\eta_{m}\right)=\eta_{m} \\ 0 & l_{\alpha}\left(\eta_{m}\right)=0\end{cases}
$$

This definition is compatible with subdivisions since we have $\tau_{m}^{\circ} \subset \eta_{m}^{\circ}$.

Now we can prove Theorem 2.17. For a sheaf $\mathcal{F}$, we denote by $G \mathcal{F}$ the canonical resolution of Godement of $\mathcal{F}$. As in the proof of Proposition 2.21, let $(K, M)$ be a semi-algebraic triangulation of the pair $(X, D)$ such that $M \triangleleft K$ and $\pi: K \rightarrow[0,1]$ be the simplicial map defined on each vertex $v$ by

$$
\pi(v)= \begin{cases}0 & v \in M \\ 1 & v \notin M\end{cases}
$$

We denote by $C_{D}$ the polytope $|C(M, K)|$, and by $i_{D}$ the inclusion of $C_{D}$ into $U$. We have the following commutative diagram with exact rows.

$$
\begin{aligned}
& 0 \rightarrow \quad \operatorname{Ker} i_{D}^{*} \rightarrow \Gamma\left(X, \mathcal{C}^{*}[-2 m]\right) \stackrel{i_{D}^{*}}{\longrightarrow} \Gamma\left(C_{D}, \mathfrak{C}^{*}[-2 m]\right) \rightarrow 0 \\
& \uparrow \alpha_{1} \quad \uparrow \alpha_{2} \quad \uparrow \alpha_{3} \\
& 0 \rightarrow C_{2 m-*}(D ; \mathbb{Z}) \rightarrow C_{2 m-*}(X ; \mathbb{Z}) \quad \rightarrow \quad C_{2 m-*}(X, D ; \mathbb{Z}) \quad \rightarrow \quad 0
\end{aligned}
$$

See Definition 2.7 for the definition of $C_{2 m-*}(D ; \mathbb{Z})$ and $C_{2 m-*}(X ; \mathbb{Z})$.

Lemma 2.23. The map $\alpha_{1}$ is a quasi-isomorphism. 
Proof. We denote by $V_{n}$ the polytope $\pi^{-1}[0,1-1 / n]$. By [14] Lemma $70.1 D$ is a deformation retract of $V_{n}$, so that the natural inclusion

$$
C_{2 m-*}(D ; \mathbb{Z}) \rightarrow C_{2 m-*}\left(V_{n} ; \mathbb{Z}\right)
$$

is a quasi-isomorphism. Since $V_{n}$ is compact, the natural map

$$
C_{2 m-*}\left(V_{n} ; \mathbb{Z}\right) \rightarrow \Gamma\left(V_{n}, \mathrm{C}^{*}[-2 m]\right)
$$

is an isomorphism. We have the assertion because $\operatorname{Ker} i_{D}^{*} \underset{n}{\lim } \Gamma\left(V_{n}, \mathrm{C}^{*}\right)$.

Since $X$ is compact, the map $\alpha_{2}$ is an isomorphism. It follows that $\alpha_{3}$ is a quasiisomorphism. Since $C_{D}$ is a deformation retract of $U$, by Proposition 2.21 the restriction map $\Gamma\left(U, \mathrm{e}^{*}\right) \rightarrow \Gamma\left(C_{D}, \mathrm{e}^{*}\right)$ is a quasi-isomorphism. Hence the natural map $\alpha_{4}$ : $C_{2 m-*}(X, D ; \mathbb{Z}) \rightarrow \Gamma\left(U, \mathrm{e}^{*}[-2 m]\right)$ is quasi-isomorphic, and the map $\beta: \Gamma\left(U, \mathrm{e}^{*}\right) \rightarrow \Gamma\left(U, G \mathrm{C}^{*}\right)$ is quasi-isomorphic since $j^{*} \mathrm{C}^{*}$ is a complex of fine sheaves. We have a commutative diagram

$$
\begin{array}{ccc}
\Gamma\left(U, G \mathcal{A \mathcal { C } ^ { * }}[-2 m]\right) & \stackrel{\iota_{1}}{\rightarrow} & \Gamma\left(U, G \mathcal{C}^{*}[-2 m]\right) \\
\uparrow r & & \uparrow \beta \\
\Gamma\left(U, \mathcal{A C}^{*}[-2 m]\right) & \rightarrow & \Gamma\left(U, \mathcal{C}^{*}[-2 m]\right) \\
\uparrow \alpha & & \uparrow \alpha_{4} \\
A C_{2 m-*}(X, D ; \mathbb{Z}) & \stackrel{\iota_{2}}{\rightarrow} & C_{2 m-*}(X, D ; \mathbb{Z})
\end{array}
$$

Since the maps $\iota_{1}, \beta \circ \alpha_{4}$ and $\iota_{2}$ are quasi-isomorphisms, the map $r \circ \alpha$ is also a quasiisomorphism. The face maps $\partial_{H_{i}}: A C_{*}(X, D ; \mathbb{Z}) \rightarrow A C_{*-2}\left(H_{i}, H_{i} \cap D ; \mathbb{Z}\right)$ induce face maps $\partial_{H_{i}}: \Gamma(U, G \mathcal{A C}[-2 m]) \rightarrow \Gamma\left(H_{i} \cap U, G \mathcal{A C}[-2(m-1)]\right)$.

Notation 2.24. For a double complex $C^{*, *}$, we denote by $s\left(C^{*, *}\right)$ the simple complex associated to $C^{*, *}$.

We see that the complex $A C^{*}(U, \mathbf{H})$ is quasi-isomorphic to the simple complex associated to the double complex

$$
\mathcal{A C}^{p, q}(U)=\underset{\sharp I=p}{\oplus} \Gamma\left(H_{I} \cap U, G \mathcal{A C}[-2(m-p)]^{q}\right) .
$$

The cohomology of the complex $s\left(\mathcal{A \mathcal { C } ^ { * , * }}(U)\right)$ is equal to $H^{*}(U, \mathbf{H} ; \mathbb{Z})$.

\section{The DUALity}

In this section we will show that the duality between the de Rham cohomology and the singular cohomology can be described via integral on admissible chains.

Definition 3.1. Let $\varphi$ be a smooth $p$-form on $X$ with logarithmic singularity along $\mathbf{H}$.

(1) Let $H_{\alpha}$ be a codimension one face of $X$. The Poincare residue of $\varphi$ at $H_{\alpha}$, which is denoted by $\operatorname{Res}_{\{\alpha\}} \varphi$, is defined as follows. If $z=0$ is a local equation of $H_{\alpha}$ and $\varphi=\frac{d z}{z} \wedge \psi+\eta$ where $\eta$ does not contain $d z$, then $\operatorname{Res}_{\{\alpha\}} \varphi=\left.\psi\right|_{H_{\alpha}}$. Note the different sign convention from the one defined in [6].

(2) For a subset $I=\left\{i_{1}<\cdots<i_{k}\right\}$ of $T$, the residue of $\varphi$ at $H_{I}$, which is denoted by $\operatorname{Res}_{I}(\varphi)$, is defined by the succession of Poincare residues

$$
\operatorname{Res}_{H_{i_{1}}} \circ \cdots \circ \operatorname{Res}_{H_{i_{k}}}(\varphi) \text {. }
$$


(3) For an element $\gamma$ of $A C_{p-\sharp I}\left(H_{I}, D \cap H_{I} ; \mathbb{Z}\right)$, the integral

$$
(2 \pi i)^{\sharp I} \int_{\gamma} \operatorname{Res}_{I}(\varphi)
$$

is denoted by $(\gamma, \varphi)$.

Proposition 3.2. Let $X$ be a smooth projective variety over $\mathbb{C}, D$ a closed subset of $X$, $U=X-D, \mathbf{H}=H_{0} \cup \cdots \cup H_{t}$ a strict normal crossing divisor on $X$ and $\varphi$ be a smooth p-form on $U$ with compact support and with logarithmic singularity along $\mathbf{H}$.

(1) For an admissible $p-\sharp I$-simplex $\sigma$ on $H_{I}$, the integral

$$
\int_{\sigma} \operatorname{Res}_{I}(\varphi)
$$

converges absolutely.

(2) Let $\gamma$ be an element of $A C_{p-\sharp I+1}\left(H_{I}, D \cap H_{I} ; \mathbb{Z}\right)$. Under the notation of Notation 3.1, we have an equality

$$
\left(\partial_{\mathbf{H}} \gamma, \varphi\right)=(\delta \gamma, \varphi)-(-1)^{\sharp I}(\gamma, d \varphi) .
$$

Remark 3.3. (1) The convergence of integrals as in the assertion (1) fails in general for $C^{\infty}$-chains. For example, consider the chain $D$ in $\mathbb{C}^{2}$ given by

$$
\left\{(x, y) \mid(x, y) \in \mathbb{R}^{2}, 1 \leq x \leq 2, e^{-\frac{1}{x-1}} \leq y \leq e^{-1}\right\} .
$$

If we set the face of $\mathbb{C}^{2}$ to be $\left\{z_{1}=0\right\} \cup\left\{z_{2}=0\right\}$, then the chain $D$ meets the faces of $\mathbb{C}^{2}$ properly, but the integral $\int_{D} \frac{d z_{1}}{z_{1}} \wedge \frac{d z_{2}}{z_{2}}$ diverges. $D$ is not $\delta$-admissible in the sense that the boundary of $D$ does not meet the face $\left\{z_{2}=0\right\}$ properly, but adding some chains in the imaginary direction we obtain a $\delta$-admissible chain.

(2) A consequence of Proposition 3.2 is that an element of $A C_{*}(U, \mathbf{H})$ defines a normal current of intersection type along $\mathbf{H}$. See [12] and [11] for the definition of normal currents of intersection type along $\mathbf{H}$.

Proof. First we consider (1). The same proof as that of [7] Theorem 4.4 works, but we need some modification. The proof of the convergence is reduced to showing "allowability" of a certain semi-algebraic set. The argument given in Section 4 of [7] should be modified as follows. We need to consider the following situation: Let $\mathbb{C}^{n} \times \mathbb{C}^{k}$ with coordinates $\left(z_{1}, \cdots, z_{n+k}\right)$. Let $H_{i}, 1=1, \cdots, n$ be the coordinate hyperplanes. A face of $\mathbb{C}^{n} \times \mathbb{C}^{k}$ is a subset of the form $H_{I}=\bigcap_{i \in I} H_{i}$ where $I$ is a subset of $\{1, \cdots, n\}$. A closed semi-algebraic subset $A$ of $\mathbb{C}^{n} \times \mathbb{C}^{k}$ is said to be admissible if for any face $H_{I}$, an inequality

$$
\operatorname{dim}\left(A \cap H_{I}\right) \leq \operatorname{dim} A-2 \sharp I
$$

holds. We need to show the following:

Claim. Let $A$ be a compact admissible semi-algebraic subset of $\mathbb{C}^{n} \times \mathbb{C}^{k}$ of dimension $h$. If $\varphi$ is an $h$-form on $\mathbb{C}^{n} \times \mathbb{C}^{k}$ of the shape

$$
\frac{d z_{1}}{z_{1}} \wedge \cdots \wedge \frac{d z_{n}}{z_{n}} \wedge \eta
$$


where $\eta$ is a smooth $(h-n)$-form on $\mathbb{C}^{n} \times \mathbb{C}^{k}$, then the integral

$$
\int_{A} \varphi
$$

converges absolutely.

We divide the complex plane $\mathbb{C}$ in to four sectors

$$
S_{i}=\left\{z=r e^{i \theta} \mid 0 \leq r<\infty,(i-2) \pi / 4 \leq \theta \leq i \pi / 4\right\}, i=0,1,2,3 .
$$

and make a coordinate change as follows: For $z=x+i y$, we set $r=|z|, \tau=y / x$. We have

$$
x=\frac{r}{\sqrt{\tau^{2}+1}}, \quad y=\frac{r \tau}{\sqrt{\tau^{2}+1}} .
$$

We have a continuous semi-algebraic map

$$
\pi: \mathbb{R}_{\geq 0} \times[-1,1] \rightarrow S_{0}, \quad(r, \tau) \mapsto(x, y) .
$$

We also have equalities

$$
\frac{d z}{z}=\frac{d r}{r}+i \frac{d \tau}{\tau^{2}+1}, \frac{d z}{z} \wedge d \bar{z}=\frac{-2(\tau+i)}{\left(\tau^{2}+1\right)^{3 / 2}} d r \wedge d \tau .
$$

The form $\varphi$ is the sum of forms of type

$$
f \frac{d z_{1}}{z_{1}} \wedge \cdots \wedge \frac{d z_{n}}{z_{n}} \wedge \bigwedge_{k \in R} d \bar{z}_{k} \wedge\left(d u_{1} \wedge \cdots \wedge d u_{h-n-|R|}\right) .
$$

Here $R$ is a subset of $\{1, \cdots, n\},\left\{u_{1}, \cdots, u_{h-n-|R|}\right\} \subset\left\{x_{n+1}, y_{n+1}, \cdots, x_{n+k}, y_{n+k}\right\}$ and $f$ is a smooth function on a neighborhood of $A$.

Writing $\tilde{\mathbb{C}}_{+}=\mathbb{R}_{\geq 0} \times[-1,1]$, let

$$
\pi=\pi_{\alpha_{1}, \cdots, \alpha_{n}} \times\left(\text { identity) }: \tilde{\mathbb{C}}_{+}^{n} \times \mathbb{C}^{k} \rightarrow\left(S_{\alpha_{1}} \times \cdots \times S_{\alpha_{n}}\right) \times \mathbb{C}^{k}\right.
$$

be the product of the maps $\pi: \tilde{\mathbb{C}}_{+} \rightarrow S_{\alpha_{i}}$ and the identity of $\mathbb{C}^{k}$, still denoted by the same letter. The pull-back of the form (3.0.2) is the sum of the forms

$$
f^{\prime} \varphi_{P, Q, R} \wedge\left(d u_{1} \wedge \cdots \wedge d u_{h-n-|R|}\right)
$$

where

$$
\varphi_{P, Q, R}=\bigwedge_{i \in P} \frac{d r_{i}}{r_{i}} \wedge \bigwedge_{j \in Q} d \tau_{j} \wedge \bigwedge_{k \in R}\left(d r_{k} \wedge d \tau_{k}\right)
$$

where $(P, Q)$ varies over partitions of $\{1, \cdots, n\}-R$, and $f^{\prime}$ is a smooth function.

Consider the map

$$
\tilde{\mathbb{C}}_{+}^{n} \times \mathbb{C}^{k} \rightarrow \mathbb{R}_{\geq 0}^{P} \times[-1,1]^{Q} \times \tilde{\mathbb{C}}_{+}^{R} \times \mathbb{R}^{h-n-|R|}
$$

given by the product of the maps

$$
\begin{array}{ll}
\tilde{\mathbb{C}}_{+}^{P} \rightarrow \mathbb{R}_{\geq 0}^{P}, & \left(r_{i}, \tau_{i}\right) \mapsto r_{i}, \\
\tilde{\mathbb{C}}_{+}^{Q} \rightarrow[-1,1]^{Q}, & \left(r_{j}, \tau_{j}\right) \mapsto \tau_{j} \\
\tilde{\mathbb{C}}_{+}^{R} \rightarrow \widetilde{\mathbb{C}}_{+}^{R}, & \text { the identity map } \\
\mathbb{C}^{k} \rightarrow \mathbb{R}^{h-n-|R|}, & \left(z_{n+1}, \cdots, z_{n+k}\right) \mapsto\left(u_{1}, \cdots, u_{h-n-|R|}\right) .
\end{array}
$$


Taking the product of these maps we obtain a map

$$
q: \tilde{\mathbb{C}}_{+}^{n} \times \mathbb{C}^{k} \rightarrow(\mathbb{R})^{P} \times(\mathbb{R})^{Q} \times\left(\mathbb{R}^{2}\right)^{R} \times \mathbb{R}^{h-n-|R|} .
$$

We need to show the absolute convergence of the integral

$$
\int_{\pi^{-1}(A)} \varphi_{P, Q, R} \wedge\left(d u_{1} \wedge \cdots \wedge d u_{h-n-|R|}\right) .
$$

Let $\varphi_{P, Q, R}^{\prime}$ be the form on $\mathbb{R}^{P} \times \mathbb{R}^{Q} \times\left(\mathbb{R}^{2}\right)^{R}$ given by the same formula as (3.0.3). Applying Proposition 2.5 [7] to the set $\pi^{-1}(A)$ and the map $q$, we are reduced to showing the absolute convergence of the integral

$$
\int_{q \pi^{-1}(A)} \varphi_{P, Q, R}^{\prime} \wedge\left(d u_{1} \wedge \cdots \wedge d u_{h-n-|R|}\right) .
$$

The argument after this is the same as that in Section 4 of [7]. Note that since the form we consider has a compact support contained in $X-D$, we can assume that $A \cap D=\emptyset$. So the argument is simpler than the case of loc. cit.

Next we prove the assertion (2). First we assume that the set $I$ is empty. The proof is in the same line as that of Theorem Theorem 4.3 [8] with some modification. Let $\mathbf{H}_{c}$ be the union of higher codimensional faces i.e.

$$
\mathbf{H}_{c}=\cup_{i \neq j}\left(H_{i} \cap H_{j}\right) .
$$

First we consider the case where $|\gamma| \cap \mathbf{H}_{c}=\emptyset$, and then prove the general case by a limit argument. So suppose that $|\gamma| \cap \mathbf{H}_{c}=\emptyset$. Let us write $\gamma=\sum a_{\sigma} \sigma$. For a codimension one face $H_{k}$, set $\gamma_{k}=\sum_{\sigma \cap H_{k} \neq \emptyset} a_{\sigma} \sigma$. Then we have

$$
\gamma=\sum_{k} \gamma_{k}+\gamma^{\prime}
$$

where $\left|\gamma^{\prime}\right| \cap \mathbf{H}=\emptyset$. since $|\gamma| \cap \mathbf{H}_{c}=\emptyset,\left|\gamma_{k}\right| \cap H_{j}=\emptyset$ for $j \neq k$, and so that $\gamma_{k} \in A C_{r}(K, D)$ for each $k$. Here we denote $r=p-\sharp I+1$ for short. It suffices to prove (2) for each $\gamma_{k}$. So we consider the case where $|\gamma| \cap \mathbf{H} \subset H_{0}=H$. For a simplex $\tau \in \gamma \cap H$, let

$$
\gamma^{(\tau)}=\sum_{\sigma \cap H=\tau} a_{\sigma} \sigma
$$

Since $K$ is a good triangulation, the intersection of each simplex $\sigma$ of $K$ with $F$ is a simplicial face of $\sigma$. So we have

$$
\gamma=\sum_{\tau \in \gamma \cap H} \gamma^{(\tau)}
$$

By Proposition 4.11 of [8], we have $\gamma^{(\tau)} \in A C_{r}(K, D)$. It suffices to prove $(2)$ for each $\gamma^{(\tau)}$. So we assume that $\gamma=\gamma^{(\tau)}$ for a simplex $\tau \in H$. By taking sufficiently fine subdivision, we can assume that $|\gamma|$ is contained in a coordinate neighborhood $V$ of $X$ on which $H$ is defined by an equation $z_{H}=0$ for a function $z_{H}$. Under the comparison isomorphism of de Rham and singular cohomology, the class of $H \cap V$ in $H_{d R, H}^{2}(V, \mathbb{C})$ is equal to $\delta\left(\frac{1}{2 \pi i} \frac{d z_{H}}{z_{H}}\right)$ where

$$
\delta: H_{d R}^{1}(V-H) \rightarrow H_{d R, H}^{2}(V)
$$


is the boundary map. For $\epsilon>0$, let $\rho_{\epsilon}: \mathbb{C} \rightarrow[0,1]$ be a $C^{\infty}$-function such that

$$
\rho_{\epsilon}(z)= \begin{cases}0 & |z|<1 / 2 \epsilon \\ 1 & |z|>\epsilon\end{cases}
$$

and set $c_{\epsilon}=\rho_{\epsilon}\left(z_{H}\right) \frac{1}{2 \pi i} \frac{d z_{H}}{z_{H}}$. Put $C=|C(H, K)|$. For $\epsilon$ sufficiently small we have $c_{\epsilon}=\frac{1}{2 \pi i} \frac{d z_{H}}{z_{H}}$ on $C$, and $\delta\left(\frac{1}{2 \pi i} \frac{d z_{H}}{z_{H}}\right)$ and $d c_{\epsilon}$ defines the same class of $H_{d R}^{2}(V, C) \simeq H_{d R}^{2}(V, V-H)=$ $H_{d R, H}^{2}(V)$. We use the form $d c_{\epsilon}$ as our Thom cocycle.

By the Stokes formula, we have

$$
\int_{\gamma} d\left(\rho_{\epsilon} \varphi\right)-\int_{\delta \gamma} \rho_{\epsilon} \varphi=0
$$

and so

$$
\int_{\gamma} d \rho_{\epsilon} \wedge \varphi=\int_{\delta \gamma} \rho_{\epsilon} \varphi-\int_{\gamma} \rho_{\epsilon} d \varphi
$$

By Lebesgue convergence theorem the right hand side of this equality converges to the right hand side of (3.0.1) as $\epsilon \rightarrow 0$. So we need to show that

$$
\lim _{\epsilon \rightarrow 0} \int_{\gamma} d \rho_{\epsilon} \wedge \varphi=2 \pi i \int_{\partial \gamma} \operatorname{Res} \varphi
$$

The rest of the argument is the same as the proof of Proposition 4.7 [8]. The limit argument which is necessary to prove the general case is the same as the one given in Section 4.4 of [8]. We consider a general case. Let $\gamma$ be an element of $A C_{p-\sharp I+1}\left(H_{I}, D \cap H_{I} ; \mathbb{Z}\right)$ with $I=\left\{\alpha_{0}<\cdots<\alpha_{k}\right\}$. If $z_{i}=0$ for $0 \leq i \leq k$ are local equations of $H_{\alpha_{i}}$, and

$$
\varphi=\frac{d z_{k}}{z_{k}} \wedge \frac{d z_{k-1}}{z_{k-1}} \wedge \cdots \wedge \frac{d z_{0}}{z_{0}} \wedge \psi+\eta
$$

where $\eta$ does not conatin $d z_{k} \wedge \cdots \wedge d z_{0}$, then $\operatorname{Res}_{I} \varphi=\left.\psi\right|_{H_{I}}$. If

$$
I^{\prime}=\left\{\alpha_{0}<\cdots<\alpha_{i-1}<\beta<\alpha_{i}<\cdots<\alpha_{k}\right\},
$$

then we have

$$
2 \pi i \int_{\partial_{H_{\beta}} \gamma} \operatorname{Res}_{\{\beta\}}\left(\operatorname{Res}_{I} \varphi\right)=\int_{\delta \gamma} \operatorname{Res}_{I} \varphi-\int_{\gamma} d\left(\operatorname{Res}_{I} \varphi\right)
$$

Since we have $\operatorname{Res}_{I^{\prime}} \varphi=(-1)^{i} \operatorname{Res}_{\{\beta\}}\left(\operatorname{Res}_{I} \varphi\right)$ and $d\left(\operatorname{Res}_{I} \varphi\right)=(-1)^{\sharp I} \operatorname{Res}_{I} d \varphi$, we have the assertion.

Theorem 3.4. The map

$$
A C^{*}(U, \mathbf{H}) \otimes A_{c}^{*}(U)(\log \mathbf{H}) \rightarrow \mathbb{C}, \quad \gamma \otimes \varphi \mapsto(-1)^{\epsilon_{1}(\gamma)}(\gamma, \varphi)
$$

is a map of complexes, and induces the duality pairing

$$
H^{*}(U, \mathbf{H} ; \mathbb{Q}) \otimes H_{d R}^{*}(X-\mathbf{H}, D ; \mathbb{C}) \rightarrow \mathbb{C} .
$$

Here the function $\epsilon_{1}(\gamma)$ is defined as follows. If $\gamma$ is on the face $H_{I}$, then $\epsilon_{1}(\gamma)=\epsilon_{1}(\operatorname{dim} \gamma, \sharp I)$ where $\epsilon_{1}(x, y)=\frac{x(x+1)}{2}+x y$. 
Proof. The fact that the above map is compatible with the differential is a consequence of Proposition 3.2. For the second assertion, first we reduce the problem to the case where $D=\emptyset$. There exists a sequence of blow-ups

$$
\pi: \widetilde{X} \rightarrow X
$$

such that $\pi^{-1}(\mathbf{H} \cup D)$ is a simple normal crossing divisor and $\pi$ induces an isomorphism $\pi^{-1}(U) \rightarrow U$.

Lemma 3.5. There is a quasi-isomorphism

$$
\pi_{*}: A C_{*}\left(\tilde{X}, \pi^{-1}(D)\right) \rightarrow A C_{*}(X, D)
$$

which is compatible with the pairing $(\bullet, \bullet):$ for $\varphi \in A_{c}^{p}(U)(\log \mathbf{H})$ and $\gamma \in A C_{p}\left(\widetilde{X}, \pi^{-1}(D)\right)$, we have $(\gamma, \varphi)=\left(\pi_{*}(\gamma), \varphi\right)$.

Proof. Let $K$ be a good triangulation of $\widetilde{X}$. For a $j$-simplex $\sigma \in K$, there is a good triangulation $K^{\prime}$ of $X$ such that $\pi(\sigma)$ is the union of the interior of some simplexes of $K^{\prime}$ by Theorem 2.1. We define $\pi_{*}(\sigma)$ to be the sum of $j$-simplexes of $K^{\prime}$ contained in $\pi(\sigma)$. The orientation of each simplex is defined by the compatibility with that of $\sigma$. By (2.2.9) we have a commutative diagram

$$
\begin{array}{ccc}
\Gamma\left(U, G \mathcal{A C}^{*}[-2 m]\right) & \stackrel{=}{\longrightarrow} & \Gamma\left(U, G \mathcal{A C} \mathcal{C}^{*}[-2 m]\right) \\
A C_{2 m-*}\left(\widetilde{X}, \pi^{-1}(D) ; \mathbb{Z}\right) & \stackrel{\pi_{*}}{\longrightarrow} & A C_{2 m-*}(X, D ; \mathbb{Z})
\end{array}
$$

From this we see that $\pi_{*}$ is a quasi-isomorphism. The compatibility of $\pi_{*}$ and the pairing $(\bullet, \bullet)$ follows from the definition.

By Lemma 3.5 we can replace $X$ resp. $\mathbf{H}$ with $\widetilde{X}$ resp. the proper transform of $\mathbf{H}$. Let $D_{j}(j=0, \cdots, s)$ be the irreducible components of $D$. We denote the index set $\{0, \cdots, s\}$ by $S$. For a subset $J$ of $S$ we denote $\bigcap_{j \in J} D_{j}$ by $D_{J}$.

Let ${ }_{d R} C^{*, *}$ be the double complex defined by

$$
{ }_{d R} C^{p q}=\underset{p=\sharp J}{\oplus} A^{q}\left(D_{J}\right)(\log \mathbf{H}), d_{1}=d_{D}, d_{1}=d_{d R} \text { (the exterior derivative) }
$$

where the differential $d_{D}$ is defined as follows: for an element $\omega=\underset{J \subset S}{\oplus} \omega_{J}$ of $d R C^{*, *}$, we have

$$
\left(d_{D} \omega\right)_{\left(\alpha_{0}<\cdots<\alpha_{k}\right)}=\sum_{i=0}^{k}(-1)^{i} \iota_{\alpha_{i}}^{*} \omega_{\left(\alpha_{0}<\cdots \widehat{\alpha_{i}} \cdots<\alpha_{k}\right)} .
$$

Here the map $\iota_{\alpha_{i}}: D_{\left(\alpha_{0}<\cdots<\alpha_{k}\right)} \rightarrow D_{\left(\alpha_{0}<\cdots \widehat{\alpha_{i}} \cdots<\alpha_{k}\right)}$ is the inclusion map.

Lemma 3.6. We have an isomorphism $H^{j}\left(A_{c}^{*}(U)(\log \mathbf{H})\right) \simeq H^{j}\left(s\left({ }_{d R} C^{*, *}\right)\right)$.

Proof. We denote by $\mathcal{A}^{*}\left(D_{J}\right)(\log \mathbf{H})$ the complex of sheaves of $C^{\infty}$ - forms on $D_{J}$ with logarithmic singularity along $\mathbf{H} .{ }_{d R} \mathrm{e}^{* * *}$ be the double complex of sheaves defined by

$$
{ }_{d R} \mathrm{C}^{p q}=\underset{p=\sharp J}{\oplus} i_{*} \mathcal{A}^{q}\left(D_{J}\right)(\log \mathbf{H})
$$

where the map $i$ is the inclusion of $D_{J}$ into $X$, and the differentials are defined in the same way as (3.0.5). Let $\pi: X \rightarrow[0,1]$ be the map defined in (2.2.8), and set $V_{n}=$ $\pi^{-1}([1 / n, 1])$ for $n \geq 1$. Let $\mathcal{A}_{V_{n}}^{*}(X)(\log \mathbf{H})$ be the complex of sheaves on $X$ of $C^{\infty}$-forms 
with $\log$ singularity along $H$ whose supports are contained in $V_{n}$. Let $\mathcal{A}_{c}^{*}(X)(\log \mathbf{H})=$ $\underset{n}{\lim } \mathcal{A}_{V_{n}}^{*}(X)(\log \mathbf{H})$. Then we have the equality $A_{c}^{*}(U)(\log \mathbf{H})=\Gamma\left(X, \mathcal{A}_{c}^{*}(X)(\log \mathbf{H})\right)$. We will show that the inclusion

$$
\mathcal{A}_{c}^{*}(U)(\log \mathbf{H}) \hookrightarrow s\left({ }_{d R} \mathrm{C}^{*, *}\right)
$$

is a quasi-isomorphism of complexes of sheaves. Then since these are complexes of fine sheaves, this inclusion induces an isomorphism of cohomology groups

$$
H^{i}\left(A_{c}^{*}(U)(\log \mathbf{H})\right) \rightarrow H^{i}\left(s\left({ }_{d R} C^{*, *}\right)\right) \simeq H^{i}(X-\mathbf{H}, \mathbf{D}, \mathbb{C}) .
$$

The problem is local, so let $x$ be a point on $D$. Near $x X$ is isomorphic to $U_{x}:=B^{J} \times B^{K}$ with $x$ the origin where $B$ is the open unit disc in $\mathbb{C}$, and $D=\left\{z_{1} \cdots z_{\sharp J}=0\right\}$. Suppose first that $J=\{1\}$. The complex

$$
\text { Cone }\left(i^{*}: \mathcal{A}^{*}\left(U_{x}\right)(\log \mathbf{H}) \rightarrow A^{*}\left(D_{1}\right)(\log \mathbf{H})\right)
$$

where $i$ is the inclusion map of $D_{1}$ into $U_{x}$, is quasi-isomorphic to Ker $i^{*}=: \mathcal{A}_{0}^{*}$. We need to show that the quotient $\mathcal{A}_{0}^{*} / \mathcal{A}_{c}^{*}(\log \mathbf{H})$ is acyclic. We proceed as the proof of Poincare Lemma. Let $F:[0,1] \times U_{x} \rightarrow U_{x}$ be the deformation retract defined by $\left(t,\left(z_{1}, \cdots, z_{1+K}\right)\right) \mapsto$ $\left((1-t) z_{1}, z_{2}, \cdots, z_{1+K}\right)$. There are maps

$$
g_{0}, g_{1}: U_{x} \rightarrow[0,1] \times U_{x}, g_{i}(p)=(i, p) .
$$

Let $h: A^{j+1}\left([0,1] \times U_{x}\right) \rightarrow A^{j}\left(U_{x}\right)$ be the map given by the integration along the fibers of the projection $[0,1] \times U_{x} \rightarrow U_{x}$. Then we have the equality

$$
g_{0}^{*}-g_{1}^{*}= \pm(d h-h d) .
$$

Suppose that $\phi$ is a cocycle of $A_{0}^{*}\left(U_{x}\right) / A_{c}^{*}\left(U_{x}\right)(\log \mathbf{H})$ i.e. $d \phi \in A_{c}^{*}\left(U_{x}\right)$. Taking $U_{x}$ sufficiently small we can assume that $d \phi=0$. By applying 3.0.6 to the form $F^{*} \phi$ we see that

$$
\phi= \pm d h F^{*} \phi .
$$

Since the restriction of $F$ to $D_{1}$ is constant with respect to $t$, we see that $h F^{*} \phi \in A_{0}^{j}\left(U_{x}\right)$. This concludes the proof for $J=\{1\}$. we can proceed by induction on $\sharp J$. Suppose the assertion holds for the case $\sharp J=k$ and consider the case $\sharp J=k+1$. We have the equality

$$
=\operatorname{Cone}\left(i_{1}^{*}: s\left(\bigoplus_{K \subset J-\{1\}} \mathcal{A}^{*}\left(D_{K}\right)\right) \rightarrow s\left(\bigoplus_{K \subset J-\{1\}} \mathcal{A}^{*}\left(D_{K \cup\{1\}}\right)\right)\right) .
$$

By induction hypothesis, This is quasi-isomorphic to the complex

$$
\text { Cone }\left(i_{1}^{*}: \mathcal{A}_{c}^{*}\left(X-\cup_{j \neq 1} D_{j}\right) \rightarrow \mathcal{A}_{c}^{*}\left(D_{1}-\cup_{j \neq 1} D_{j}\right)\right) .
$$

By the same argument as in the proof of the case where $\sharp J=1$, we can show that this complex is quasi-isomorphic to $\mathcal{A}_{c}^{*}\left(U_{x}\right)$.

The complex $C_{*}(X) / C_{*}(D)$ is quasi-isomorphic to the simple complex associated to the double complex $C(1)_{*, *}$ defined by

$$
C(1)_{a, b}=\underset{J \subset S, \sharp J=a}{\bigoplus} C_{b}\left(D_{J}\right) .
$$


Here the second differential is $\delta$, the topological boundary, and the first differential $\partial_{D}$ is defined by

$$
\partial_{D}\left(\gamma_{\left(\alpha_{0}<\cdots<\alpha_{p}\right)}\right)=\sum_{i=0}^{p}(-1)^{i} \iota_{\alpha_{i}}\left(\gamma_{\left(\alpha_{0}<\cdots<\alpha_{p}\right)}\right)
$$

where $\iota_{\alpha_{i}}: H_{\left\{\left(\alpha_{0}, \cdots, \alpha_{p}\right)\right\}} \hookrightarrow H_{\left\{\alpha_{0}, \cdots, \widehat{\alpha_{i}}, \cdots, \alpha_{p}\right\}}$ is the inclusion map. By Proposition [2.8, $C(1)_{*, *}$ is quasi-isomorphic to the complex $C(2)_{*, *}$ defined by

$$
C(2)_{a, b}=\underset{J \subset S, \sharp J=a}{\oplus} A C_{b}\left(D_{J}\right) .
$$

Hence the complex $A C^{*}(U, \mathbf{H})$ is quasi-isomorphic to the simple complex $s\left(C(3)^{*, *}\right)$ associated to the double complex $C(3)^{* * *}$ defined by

$$
\left.C(3)^{p, q}=\underset{\substack{I \subset T, \sharp I=p \\ J \subset S, \sharp J+i=2 m-2 p-q}}{\bigoplus} A C_{i}\left(H_{I} \cap D_{J}\right)\right), d_{1}=\partial_{\mathbf{H}}, d_{2}=\partial_{D}+(-1)^{\sharp J} \delta .
$$

The differential of $s\left(C(3)^{*, *}\right)$ is equal to

$$
\partial_{\mathbf{H}}+(-1)^{\sharp I} \partial_{D}+(-1)^{\sharp I+\sharp J} \delta
$$

on $A C_{*}\left(H_{I} \cap D_{J}\right)$. Let ${ }_{t o p} C^{p, q}$ be the double complex defined by

$$
{ }_{t o p} C^{p q}=\underset{p=-\sharp J, q=2 m-\sharp I-i}{\oplus} A C_{i}\left(H_{I} \cap D_{J}\right), d_{1}=\partial_{D}, d_{2}=\partial_{\mathbf{H}}+(-1)^{\sharp I} \delta
$$

The map $s\left(C(3)^{*, *}\right) \rightarrow s\left({ }_{t o p} C^{*, *}\right)$ defined by $\gamma \mapsto(-1)^{\sharp I \sharp J} \gamma$ for $\gamma \in A C_{*}\left(H_{I} \cap D_{J}\right)$ is an isomorphism of complexes. By Proposition 3.2, the pairing of Notation 3.1 (2) induces a pairing

$$
s\left({ }_{t o p} C^{*, *}\right) \otimes s\left({ }_{d R} C^{*, *}\right) \rightarrow \mathbb{C}[-2 m], \gamma \otimes \varphi \mapsto(-1)^{\epsilon_{1}(\gamma)+\epsilon_{2}(\gamma)}(\gamma, \varphi)
$$

which is a map of complexes. Here the target is the complex $\mathbb{C}$ concentrated in degree $2 m$, and the function $\epsilon_{2}$ is defined as follows. If $\gamma$ is on $D_{J} \cap H_{I}$, then $\epsilon_{2}(\gamma)=\epsilon_{2}(\operatorname{dim} \gamma-\sharp I, \sharp J)$ where $\epsilon_{2}(x, y)=x y+\frac{y(y+1)}{2}$. We need to show that this pairing induces perfect pairing on cohomology. The above pairing induces a map of double complexes

$$
{ }_{t o p} C^{p q} \rightarrow\left({ }_{d R} C^{-p 2 m-q}\right)^{\vee}
$$

which is compatible with the differentials up to sign. There are spectral sequences

$$
{ }_{\text {top }} E_{0}^{p q}=\underset{p=-\sharp J, q=2 m-\sharp I-j}{\oplus} A C_{j}\left(H_{I} \cap D_{J}\right) \Rightarrow H^{p+q}\left(s\left({ }_{\text {top }} C^{*, *}\right)\right)
$$

and

$$
{ }_{d R} E_{0}^{p q}=\underset{p=\sharp J}{\bigoplus^{q}}\left(D_{J}\right)(\log \mathbf{H}) \Rightarrow H^{p+q}\left(s\left({ }_{d R} C^{*, *}\right)\right) .
$$

The above pairing induces a map of spectral sequences

$$
{ }_{\text {top }} E_{r}^{p q} \rightarrow\left({ }_{d R} E_{r}^{-p 2 m-q}\right)^{\vee}
$$

compatible with the differentials up to sign. So it suffices to show that the map

$$
{ }_{\text {top }} E_{1}^{p q} \rightarrow\left({ }_{d R} E_{1}^{-p 2 m-q}\right)^{\vee}
$$

is an isomorphism for each $(p, q)$. This follows from the assertion of Theorem 3.4 for each $D_{J}$ since

$$
d R E_{1}^{-p 2 m-q}=\underset{p=-\sharp J}{\bigoplus} H_{d R}^{2 m-q}\left(D_{J}-\mathbf{H}\right)
$$


and

$$
{ }_{\text {top }} E_{1}^{p q}=\underset{p=-\sharp J}{\oplus} H^{2(m+p)-(2 m-q)}\left(D_{J}, \mathbf{H} ; \mathbb{Z}\right) .
$$

Until the end of the proof of Theorem 3.4 we assume that $D=\emptyset$.

Lemma 3.7. (1) Let $K$ be a complex and $L$ be a full subcomplex of $K$. Let $K^{\prime}$ be a derived subdivision of $K \bmod L \cup C(L, K)$. Then $\left|N\left(C(L, K), K^{\prime}\right)\right|$ is a regular neighborhood of $|C(L, K)|$.

(2) Suppose that $L$ is a full subcomplex of $K$. If $K^{\prime}$ is a subdivision of $K$ inducing a subdivision $L^{\prime}$ of $L$, then we have $L^{\prime} \triangleleft K^{\prime}$.

Proof. (1). Since $L$ is a full subcomplex of $K$, we have $L=C(C(L, K), K)$, and the assertion follows from the definition of a regular neighborhood.

(2). This is [15] Lemma $3.3(\mathrm{~b})$.

Lemma 3.8. Let $K$ be a complex and let $L_{1}$ and $L_{2}$ subcomplexes of $K$ such that $L_{2}$ and $L_{1} \cup L_{2}$ are full subcomplexes of $K$. Then $N\left(L_{1}, K\right) \cap L_{2}$ equals $N\left(L_{1} \cap L_{2}, L_{2}\right)$.

Proof. Suppose that $\tau, \eta \in K$ such that $\tau \prec \eta, \tau \in L_{2}$ and $\eta \cap L_{1} \neq \emptyset$. By the assumption $\sigma:=\eta \cap\left(L_{1} \cup L_{2}\right)$ is a face of $\eta$. If $\sigma \in L_{2}$ we are done since $\tau \prec \sigma$ and $\sigma \cap L_{1} \neq \emptyset$. If $\sigma \in L_{1}$ there is a vertex $v \in \sigma \cap L_{2}$ since $\eta \cap L_{2} \neq \emptyset$. The simplex spanned by $\tau$ and $v$ is in $L_{2}$ since $L_{2}$ is a full subcomplex of $K$.

Proposition 3.9. Let $(K, L)$ be a good triangulation of the pair $(X, \mathbf{H})$ such that $|N(L, K)|$ is a regular neighborhood of $\mathbf{H}$ in $X$. Then the natural inclusion $C_{*}(C(L, K) ; \mathbb{Q}) \rightarrow A C^{2 m-*}(X, \mathbf{H} ; \mathbb{Q})$ is a quasi-isomorphism.

Proof. Recall that we set $\mathbf{H}=H_{0} \cup \cdots \cup H_{t}$. We proceed by induction on $t$. When $t<0$ i.e. $\mathbf{H}$ is empty there is nothing to prove. Assume that the assertion holds for $t=k$ and consider the case where $t=k+1$. We denote $H_{0} \cup \cdots \cup H_{k}$ by $F_{1}$ and $H_{k+1}$ by $F_{2}$. We set $L_{1}=K \cap F_{1}$ and $L_{2}=K \cap F_{2}$. Since $K$ is a good triangulation, we have $L_{1} \triangleleft K, L_{2} \triangleleft K$ and $L_{1} \cup L_{2} \triangleleft K$.

For a complex $K$ and a subcomplex $L$ of $K$, a derived subdivision $K^{\prime}$ of $K \bmod L \cup$ $C(L, K)$ is called a derived of $K$ near $L$. cf. 3.5 [15]. If $L \triangleleft K$, then the polytope $\left|N\left(L, K^{\prime}\right)\right|$ is a regular neighborhood of $|L|$ in $|K|$.

Lemma 3.10. Let $K^{\prime}$ be a derived of $K$ near $L_{1}$, and put $N_{1}=N\left(L_{1}, K^{\prime}\right)$ and $C_{1}=$ $C\left(L_{1}, K^{\prime}\right)$. Let $\mu_{C_{1}} \in C_{*}\left(C_{1}, \partial C_{1}\right)$ be the fundamental chain of $C_{1}$. Then the map

$$
\cap \mu_{C_{1}}, C^{*}\left(C_{1}, \dot{N}_{1} ; \mathbb{Q}\right) \rightarrow C_{*}\left(C_{1}, \mathbb{Q}\right)
$$

is quasi-isomorphic. Here we denote $N_{1} \cap C_{1}$ by $\dot{N}_{1}$.

Proof. By Lemma 3.7 and Proposition 3.10 [15] $C_{1}:=C\left(C\left(L_{1}, K\right), K^{\prime}\right)$ is a manifold with the boundary $\dot{N}_{1}$. The assertion follows from Lefschetz duality Theorem 3.43 [9].

Notation 3.11. Let $K$ be a complex and $L$ be a subcomplex of $K$. If $K^{\prime}$ is a subdivision of $K$, the subdivision of $L$ induced by $K^{\prime}$ is denoted by $L^{\prime}$. Similar notations will be used for other superscripts than'. 
Let $\operatorname{sd} C_{1}$ be the barycentric subdivision of $C_{1}$. We see that

$$
\operatorname{sd}\left(L_{2}^{\prime} \cap C_{1}\right) \cup \operatorname{sd} \dot{N}_{1} \triangleleft \operatorname{sd} C_{1} .
$$

Let $C_{1}^{(2)}$ be a derived of $\operatorname{sd} C_{1}$ near $\operatorname{sd}\left(L_{2}^{\prime} \cap C_{1}\right)$, and set $N_{2}:=N\left(\operatorname{sd}\left(L_{2}^{\prime} \cap C_{1}\right), C_{1}^{(2)}\right)$. A simplex $\sigma$ of $N_{1}$ which meets $L_{1}$ but not contained in $L_{1}$ is of the form $\tau_{1} * \tau_{2}$ where $\tau_{1} \in L_{1}$ and $\tau_{2} \cap L_{1}=\emptyset$, since $L_{1} \triangleleft K$. In $\dot{N}_{1}{ }^{(2)} \tau_{2}$ is replaced with a complex $T$. Let $N_{1}{ }^{(2)}$ be the subdivision of $N_{1}$ obtained by replacing each such simplex $\sigma$ with $\tau_{1} * T$. The union $K^{(2)}:=C_{1}^{(2)} \cup N_{1}^{(2)}$ is a subdivision of $K$, and we have $N_{1}^{(2)} \cup N_{2}=N\left(L_{1} \cup L_{2}^{(2)}, K^{(2)}\right)$.
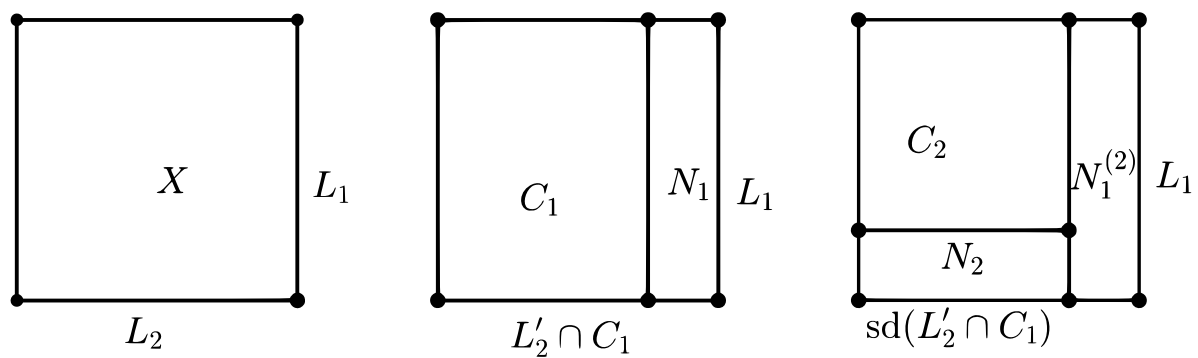

Lemma 3.12. (1) Let $C_{2}:=C\left(\operatorname{sd}\left(L_{2}^{\prime} \cap C_{1}\right), C_{1}^{(2)} ; \mathbb{Q}\right)$ and set $\mu_{C_{2}} \in C_{*}\left(C_{2}, \partial C_{2} ; \mathbb{Q}\right)$ be the fundamental cycle of $C_{2}$. Then the map

$$
\cap \mu_{C_{2}}: C^{*}\left(K^{(2)},\left(N_{1}\right)^{(2)} \cup N_{2} ; \mathbb{Q}\right) \rightarrow C_{*}\left(C_{2}, \mathbb{Q}\right)
$$

is quasi-isomorphic.

(2) The map

$$
i^{*}: C^{*}\left(N_{2}, \dot{N}_{1}^{(2)} \cap N_{2} ; \mathbb{Q}\right) \rightarrow C^{*}\left(\operatorname{sd}\left(L_{2} \cap C_{1}\right),\left(\dot{N}_{1} \cap L_{2}\right)^{(2)} ; \mathbb{Q}\right)
$$

given by the restriction is a quasi-isomorphism.

Proof. (1). By the construction we have

$$
C^{*}\left(K^{(2)},\left(N_{1}\right)^{(2)} \cup N_{2} ; \mathbb{Q}\right)=C^{*}\left(C_{2}^{(2)}, \partial C_{2}^{(2)} ; \mathbb{Q}\right) .
$$

The assertion follows from Lefschetz duality Theorem 3.43 [9].

(2). By (3.0.8) and Lemma $3.7(2)$ we see that

$$
\operatorname{sd}\left(L_{2}^{\prime} \cap C_{1}\right) \cup \dot{N}_{1}^{(2)} \triangleleft C_{1}^{(2)} .
$$

Hence by Lemma 3.8 we see that

$$
\dot{N}_{1}^{(2)} \cap N_{2}=N\left(\operatorname{sd}\left(L_{2} \cap \dot{N}_{1}\right), \dot{N}_{1}{ }^{(2)}\right)
$$

and so $\left|\dot{N}_{1}^{(2)} \cap N_{2}\right|$ is a regular neighborhood of $F_{2} \cap\left|\dot{N}_{1}\right|$ in $\left|\dot{N}_{1}\right|$. The assertion follows from this, since a polytope is a deformation retract of its regular neighborhood . 
Let $K^{(3)}$ be a derived of $K^{(2)}$ near $L_{1}$, and set $\mathcal{N}_{1}:=N\left(L_{1}, K^{(3)}\right)$. We see that $\left|\mathcal{N}_{1}\right|$ is a regular neighborhood of $F_{1}$ in $X$. We denote $\mathcal{C}_{1}=C\left(L_{1}, K^{(3)}\right)$. Since $\left|N_{1}\right|$ and $\left|\mathcal{N}_{1}\right|$ are regular neighborhoods of $F_{1}$, the map

$$
C^{*}\left(K^{(3)}, N_{1} ; \mathbb{Q}\right) \rightarrow C^{*}\left(K^{(3)}, \mathcal{N}_{1} ; \mathbb{Q}\right)
$$

induced by the inclusion $\mathcal{N}_{1} \rightarrow N_{1}$ is quasi-isomorphic. By Lemma 3.8 we have $N_{1} \cap L_{2}^{\prime}=$ $N\left(L_{1} \cap L_{2}^{\prime}, L_{2}^{\prime}\right)$ and $\mathcal{N}_{1} \cap L_{2}^{(3)}=N\left(L_{1} \cap L_{2}^{(3)}, L_{2}^{(3)}\right)$, so that $\left|N_{1} \cap L_{2}^{\prime}\right|$ and $\left|\mathcal{N}_{1} \cap L_{2}^{(3)}\right|$ are regular neighborhoods of $F_{1} \cap F_{2}$ in $F_{2}$. Hence the natural map

$$
C^{*}\left(L_{2}^{(3)},\left(N_{1} \cap L_{2}\right)^{(3)} ; \mathbb{Q}\right) \rightarrow C^{*}\left(L_{2}^{(3)},\left(\mathcal{N}_{1} \cap L_{2}\right)^{(3)} ; \mathbb{Q}\right)
$$

is also quasi-isomorphic.

Lemma 3.13. There exists a subdivision $K^{(4)}$ of $K^{(3)}$ which satisfies the following: There is a subcomplex $\widetilde{C}_{0}$ resp. $\widetilde{C}$ of $C_{*}\left(C_{2}^{(4)}, \mathbb{Q}\right)$ resp. of $C_{*}\left(\mathcal{C}_{1}{ }^{(4)}, \mathbb{Q}\right)$ which satisfies the following conditions.

(1) The inclusion of $C_{2}^{(4)}$ into $\mathcal{C}_{1}^{(4)}$ induces an inclusion of $\widetilde{C}_{0}$ into $\widetilde{C}$.

(2) The inclusion $i_{0}: \widetilde{C}_{0} \rightarrow C_{*}\left(C_{2}^{(4)}, \mathbb{Q}\right)$ resp. $i_{2}: \widetilde{C} \rightarrow C_{*}\left(\mathcal{C}_{1}{ }^{(4)}, \mathbb{Q}\right)$ is a quasiisomorphism.

(3) For each chain $\gamma \in \widetilde{C},|\gamma|$ and $|\delta \gamma|$ meet all faces properly.

Proof. For each $j \geq 0$ take a basis of $H_{j}\left(C_{2}^{(3)}, \mathbb{Q}\right)$ as follows. First take a basis $\mathbf{k}_{j}=\left\{k_{j, \alpha}\right\}_{\alpha}$ of the kernel of the map $\left(\iota_{2}\right)_{*}: H_{j}\left(C_{2}^{(3)}, \mathbb{Q}\right) \rightarrow H_{j}\left(C_{1}^{(3)}, \mathbb{Q}\right)$ induced by the inclusion. Extend this to a basis $\mathbf{k}_{j} \cup \mathbf{l}_{j}=\left\{k_{j, \alpha}\right\}_{\alpha} \cup\left\{l_{j, \beta}\right\}_{\beta}$ of $H_{j}\left(C_{2}^{(3)}, \mathbb{Q}\right)$. Take a lift $\mathbf{K}_{j} \cup \mathbf{L}_{j}=$ $\left\{K_{j, \alpha}\right\}_{\alpha} \cup\left\{L_{j, \beta}\right\}_{\beta}$ of $\mathbf{k}_{j} \cup \mathbf{l}_{j}$ in $C_{j}\left(C_{2}^{(3)}, \mathbb{Q}\right)$ and define $\widetilde{C_{0}}$ to be the complex generated by them for each $j$. Extend $\iota_{2}\left(\mathbf{l}_{j}\right)$ to a basis $\iota_{2}\left(\mathbf{l}_{j}\right) \cup \mathbf{m}_{j}=\left\{\iota_{2}\left(l_{j, \beta}\right)\right\}_{\beta} \cup\left\{m_{j, \xi}\right\}_{\xi}$ of $H_{j}\left(C_{1}^{(3)}, \mathbb{Q}\right)$. Take a lift $\mathbf{M}_{j}=\left\{M_{j, \xi}\right\}_{\xi}$ of $\mathbf{m}_{j}$ in $C_{j}\left(C_{1}^{(3)}, \mathbb{Q}\right)$. Let $\boldsymbol{\Gamma}_{j}=\left\{\Gamma_{j, \alpha}\right\}_{\alpha}$ be chains in $C_{j+1}\left(C_{1}^{(3)}, \mathbb{Q}\right)$ such that $\delta \Gamma_{j, \alpha}=\iota_{2}\left(K_{j, \alpha}\right)$ for each $\alpha$. Set $\mathcal{X}=\left\{\cup_{j, \alpha} \iota_{2}\left(\left|K_{j, \alpha}\right|\right)\right\} \cup\left\{\cup_{j, \beta} \iota_{2}\left(\left|L_{j, \beta}\right|\right)\right\} \cup\left\{\cup_{j, \xi}\left|M_{j, \xi}\right|\right\}$ and $X_{0}=\left\{\cup_{j, \alpha} \iota_{2}\left(\left|K_{j, \alpha}\right|\right)\right\} \cup\left\{\cup_{j, \beta} \iota_{2}\left(\left|L_{j, \beta}\right|\right)\right\}$. By the construction $X$ does not meet $\left|\mathcal{N}_{1}\right|$ so that $X-X_{0} \subset\left|\stackrel{\circ}{\complement}_{1}\right|$. Hence by Theorem $2.20 X$ can be moved in $\left|\stackrel{\circ}{\complement}_{1}\right|$ by an isotopy which keeps $X_{0}$ fixed, so that $h_{1}\left(X-X_{0}\right)$ meet $F_{2}$ properly. Similarly the chains $\Gamma_{j, \alpha}$ can be moved keeping $\left\{\cup_{j} \iota_{2}\left(\left|\mathbf{K}_{j}\right|\right)\right\} \cup\left\{\underset{j}{\cup} \iota_{1}\left(\left|\mathbf{L}_{j}\right|\right)\right\}$ fixed, so that $h_{1}\left(\left|\Gamma_{j, \alpha}\right|\right)$ meet $F_{2}$ properly. Let $\widetilde{C}$ be the complex generated in degree $j$ by $\left\{h_{1}\left(\Gamma_{j-1, \alpha}\right)\right\}_{\alpha},\left\{\iota_{2}\left(K_{j, \alpha}\right)\right\}_{\alpha},\left\{\iota_{2}\left(L_{j, \beta}\right)\right\}_{\beta}$ and $\left\{h_{1}\left(M_{j, \xi}\right)\right\}_{\xi}$. 
We take a good ordering of $K^{(4)}$ with respect to $L_{2}^{(4)}$. We have the following diagram.

$$
\begin{array}{ccccc}
C^{*}\left(K, N_{1} \cup N_{2}\right) & \stackrel{\iota_{0}}{\longrightarrow} & \text { Cone }\left(C^{*}\left(K, N_{1}\right)\right. & \stackrel{\text { res }}{\longrightarrow} & \left.C^{*}\left(N_{2}, N_{1} \cap N_{2}\right)\right) \\
\downarrow= & & \mathrm{n}_{2} & & \downarrow \mathrm{n}_{1} \\
C^{*}\left(K, N_{1} \cup N_{2}\right) & \stackrel{\iota_{1}}{\longrightarrow} & \text { Cone }\left(C^{*}\left(K, \mathcal{N}_{1}\right)\right. & \stackrel{i^{*}}{\longrightarrow} & \left.C^{*}\left(L_{2}, \mathcal{N}_{1} \cap L_{2}\right)\right) \\
\downarrow \cap \mu_{C_{2}} & & \downarrow \cap \mu_{\mathcal{C}_{1}} & H & \downarrow \mu_{L_{2}} \\
C_{*}\left(C_{2}\right) & \stackrel{\iota_{2}}{\longrightarrow} & \text { Cone }\left(C_{*}\left(\mathcal{C}_{1}\right)\right. & \stackrel{\cap T_{F_{2}}}{\longrightarrow} & \left.C_{*}\left(L_{2} \cap \mathcal{C}_{1}\right)\right) \\
\uparrow i_{0} & & \uparrow i_{2} & & \uparrow= \\
\widetilde{C_{0}} & \stackrel{\iota_{3}}{\longrightarrow} & \text { Cone }(\widetilde{C} & \stackrel{\cap T_{F_{2}}}{\longrightarrow} & \left.C_{*}\left(L_{2} \cap \mathcal{C}_{1}\right)\right) \\
\downarrow i_{0} & & \downarrow & & \\
C_{*}\left(C_{2}\right) & \stackrel{\iota_{4}}{\longrightarrow} & \text { Cone }\left(A C^{\prime}\left(X, F_{1}\right)\right. & \stackrel{\cap T_{F_{2}}}{\longrightarrow} & \left.A C\left(F_{2}, F_{1} \cap F_{2}\right)\right)
\end{array}
$$

Here we omitted the superscript ${ }^{(4)}$ which should be put on every simplicial complex appearing in the diagram, and the coefficient $\mathbb{Q}$. The chain $\mu_{C_{2}}$ resp. $\mu_{\mathrm{C}_{1}}$ resp. $\mu_{L_{2}}$ is the fundamental cycle of $C_{2}$ resp. $\mathcal{C}_{1}$ resp. $L_{2}$. By Lefschetz duality Theorem 3.43 [9], the maps $\cap \mu_{*}$ for $*=C_{2}, \mathcal{C}_{1}$ and $L_{2}$ are quasi-isomorphic. The map res is the restriction. The map $\mathrm{n}_{2}$ is the inclusion, and $\mathrm{n}_{1}$ is the composition of $i^{*}$ of Lemma 3.12 (2) and the map in (3.0.9). The maps $\iota_{1}$ through $\iota_{5}$ are induced by inclusions. All the squares except for the one with an $H$ in it is commutative. The one with $H$ is homotopy commutative. The complex $A C^{\prime}\left(X, F_{1}\right)$ is defined as follows: Let $A C\left(X, F_{1}\right)$ be the complex defined as in Definition 2.16 for the face $F_{1}=H_{0} \cup \cdots \cup H_{k} . A C^{\prime}\left(X, F_{1}\right)$ is the subcomplex of $A C\left(X, F_{1}\right)$ which consists of the chains $\gamma$ such that $|\gamma|$ and $|\delta \gamma|$ meet $F_{2}$ properly. By Theorem 2.20 $A C^{\prime}\left(X, F_{1}\right)$ is quasi-isomorphic to $A C\left(X, F_{1}\right)$. The map $\iota_{0}$ is quasi-isomorphic. Since $\mathrm{n}_{1}$ and $\mathrm{n}_{2}$ are quasi-isomorphic, $\iota_{1}$ is quasi-isomorphic. The maps $\iota_{2}$ and $\iota_{3}$ are quasi-isomorphic by similar reasons.

We have the following commutative diagram.

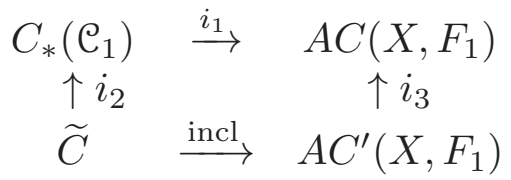

Here the maps $I_{1}$ and $i_{3}$ are natural inclusions. The map $i_{1}$ is a quasi-isomorphism by the induction hypothesis, and the maps $i_{2}$ and $i_{3}$ are quasi-isomorphic by the construction. Hence the map incl is also quasi-isomorphic. Since the map $\iota_{5}$ is quasi-isomorphic by the induction hypothesis, the map $\iota_{4}$ is a quasi-isomorphism. The complex

$$
\text { Cone }\left(A C^{\prime}\left(X, F_{1}\right) \stackrel{\cap T_{F_{2}}}{\longrightarrow} A C\left(F_{2}, F_{1} \cap F_{2}\right)\right)
$$

is $A C(X, \mathbf{H})$ for $t=k+1$. Let $\left(K^{(2)}\right)^{\prime}$ be a derived of $K^{(2)}$ near $L_{1} \cup L_{2}^{(2)}$. Set $\overline{N_{2}}=$ $N\left(L_{1} \cup L_{2},\left(K^{(2)}\right)^{\prime}\right)$ and $\overline{C_{2}}=C\left(L_{1} \cup L_{2},\left(K^{(2)}\right)^{\prime}\right)$. Then by Lemma 3.7 and the assumption that $L_{1} \cup L_{2} \triangleleft K,\left|\overline{C_{2}}\right|$ is a regular neighborhood of $\left|C_{2}\right|$. It follows that the map of complexes

$$
C_{*}\left(C_{2}^{(2)}\right) \rightarrow C_{*}\left(\overline{C_{2}}\right)
$$

induced by the inclusion $C_{2}^{(2)} \rightarrow \overline{C_{2}}$ is a quasi-isomorphism. By the uniqueness of regular neighborhood Theorem 3.24 [15], we have the assertion. 
We have the following commutative diagram.

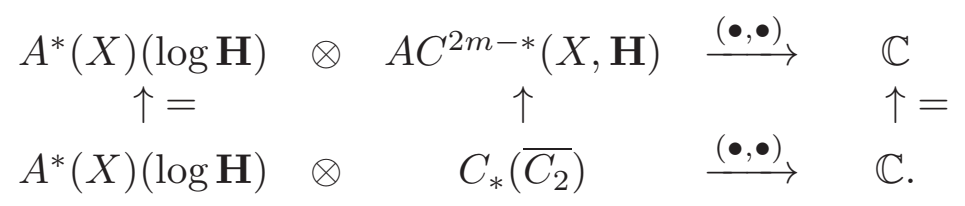

Here the map $(\bullet, \bullet)$ is the one defined in Notation 3.1. In this diagram all the vertical arrows are quasi-isomorphic, and the bottom line of this diagram induces the duality pairing

$$
H_{d R}^{k}(X-\mathbf{H}) \otimes H_{k}\left(\left|\overline{C_{2}}\right| ; \mathbb{C}\right) \rightarrow \mathbb{C}
$$

by de Rham Theorem. Hence the pairing of the first line also induces perfect pairings on cohomology groups.

\section{The Abel-Jacobi map for higher Chow Cycles}

In the following we consider the case where $X=Y \times\left(\mathbb{P}^{1}\right)^{n}$ with $Y$ a smooth projective complex variety of dimension $m$. Let $z_{i}(i=1, \cdots, n)$ be the affine coordinates of $\left(\mathbb{P}^{1}\right)^{n}$. The faces are intersections of subvarieties $\left\{z_{i}=0\right\}$ and $\left\{z_{i}=\infty\right\}$ for $i=1, \cdots, n$. The divisor at infinity $D$ equals $\bigcup_{i=1}^{n}\left\{z_{i}=1\right\}$. The complement $\left(\mathbb{P}^{1}\right)^{n}-D$ is denoted by $\square^{n}$, and the union of all faces of $\square^{n}$ is denoted by $\partial \square^{n}$. For $1 \leq i \leq n$ and $\beta \in\{0, \infty\}$, let $H_{i, \beta}$ be the face $\left\{z_{i}=\alpha\right\}$. We define the ordering of codimension one faces of $\square^{n}$ by

$$
H_{1,0}<H_{1, \infty}<H_{2, \infty}<H_{2,0}<H_{3,0}<H_{3, \infty}<H_{4, \infty}<\cdots .
$$

Here the numbering starts from 0 . We denote the set of the indices $\{(1,0),(1, \infty), \cdots,(n, \infty)\}$ of codimension one faces of $\square^{n}$ by $T$. For a subset $I$ of $T$, the face $\bigcap_{i \in I} H_{i}$ is denoted by $H_{I}$. The group $G_{n}=\{ \pm 1\}^{n} \rtimes S_{n}$ acts naturally on $\square^{n}$ as follows. The subgroup $\{ \pm 1\}^{n}$ acts by the inversion of the coordinates $z_{i}$, and the symmetric group $S_{n}$ acts by permutation of $z_{i}$ 's. Let sign : $G_{n} \rightarrow\{ \pm 1\}$ be the character which sends $\left(\epsilon_{1}, \cdots, \epsilon_{n} ; \sigma\right)$ to $\epsilon_{1} \cdots \epsilon_{n} \cdot \operatorname{sign}(\sigma)$. The idempotent Alt $=\operatorname{Alt}_{n}:=\left(1 /\left|G_{n}\right|\right) \sum_{g \in G_{n}} \operatorname{sign}(g) g$ in the group ring $\mathbb{Q}\left[G_{n}\right]$ is called the alternating projector. For a $\mathbb{Q}\left[G_{n}\right]$-module M, the submodule

$$
M^{\text {alt }}=\{\alpha \in M \mid \text { Alt } \alpha=\alpha\}=\operatorname{Alt}(M)
$$

is called the alternating part of $M$. The product $\square^{n} \times Y$ resp. $\partial \square^{n} \times Y$ will be written $\square^{n} Y$ resp. $\partial \square_{Y}^{n}$ for short. We recall the definition of the cubical version of higher Chow groups and of the Abel-Jacobi map. We denote by $z^{p}(X, n)$ the free $\mathbb{Q}$-vector space generated by subvarieties of $\square^{n} Y$ meeting faces properly. The differential is defined to be

$$
\partial_{\square}=\sum_{j=1}^{n}(-1)^{j-1}\left(\partial_{0}^{j}-\partial_{\infty}^{j}\right): z^{p}(Y, n)^{\text {alt }} \rightarrow z^{p}(Y, n-1)^{\text {alt }}
$$

Here the map $\partial_{i}^{0}$ resp. $\partial_{i}^{\infty}$ is the face map of the face $H_{i, 0}$ resp. $H_{i, \infty}$. By definition

$$
C H^{p}(Y, n)=H_{n}\left(z^{p}(Y, *)^{\text {alt }}\right) .
$$

Lemma 4.1. Let $Z$ be an element of $z^{p}(Y, n)^{\text {alt }}$.

(1) $H_{|Z|}^{j}\left(\square_{Y}^{n}, \partial \square_{Y}^{n} ; \mathbb{Q}(p)\right)=0$ for $j<2 p$. 
(2) There is an isomorphism

$H_{|Z|}^{2 p}\left(\square_{Y}^{n}, \partial \square_{Y}^{n} ; \mathbb{Q}(p)\right)=\bigcap_{i} \operatorname{Ker}\left(\partial_{i}: H_{|Z|}^{2 p}\left(\square_{Y}^{n}, \mathbb{Q}(p)\right) \rightarrow H_{|Z| \cap H_{i Y}}^{2 p}\left(H_{i Y}, \mathbb{Q}(p)\right)\right)$

Here the intersection on the right hand side is taken over all codimension one faces of $\square^{n}$.

Proof. There is a spectral sequence

$$
E_{1}^{a b}=\underset{\sharp I=a}{\oplus} H_{|Z| \cap H_{I}}^{b}\left(H_{I} ; \mathbb{Q}(p)\right) \Rightarrow H_{|Z|}^{a+b}\left(\square_{Y}^{n}, \partial \square_{Y}^{n} ; \mathbb{Q}(p)\right) .
$$

Since $|Z|$ meets every face properly, we have $H_{|Z| \cap H_{I}}^{b}\left(H_{I} ; \mathbb{Q}(p)\right)=0$ for $j<2 p$ and

$$
\begin{aligned}
& H_{|Z|}^{2 p}\left(\square_{Y}^{n}, \partial \square_{Y}^{n} ; \mathbb{Q}(p)\right) \\
= & E_{2}^{02 p} \\
= & \left.\operatorname{Ker}\left(\oplus_{i} \partial_{i}: H_{|Z|}^{2 p}\left(\square_{Y}^{n}, \mathbb{Q}(p)\right) \rightarrow \underset{i}{\oplus_{|Z| \cap H_{i Y}}} H_{i Y}^{2 p}, \mathbb{Q}(p)\right)\right) .
\end{aligned}
$$

The assertion follows from this.

There is a localization sequence of mixed Hodge structures

$$
\begin{aligned}
& 0 \rightarrow H^{2 p-1}\left(\square_{Y}^{n}, \partial \square_{Y}^{n} ; \mathbb{Q}(p)\right) \stackrel{\beta}{\rightarrow} H^{2 p-1}\left(\square_{Y}^{n}-|Z|, \partial \square_{Y}^{n}-|Z| ; \mathbb{Q}(p)\right) \\
& \rightarrow H_{|Z|}^{2 p}\left(\square_{Y}^{n}, \partial \square_{Y}^{n} ; \mathbb{Q}(p)\right) \stackrel{\iota}{\rightarrow} H^{2 p}\left(\square_{Y}^{n}, \partial \square_{Y}^{n} ; \mathbb{Q}(p)\right) .
\end{aligned}
$$

As we will see shortly, there is an isomorphism $H^{j}\left(\left(\square_{Y}^{n}, \partial \square_{Y}^{n} ; \mathbb{Q}(p)\right) \simeq H^{j-n}(Y, \mathbb{Q}(p))\right.$. The map $\beta$ induces an isomorphism

$$
\begin{aligned}
J^{p, n}(Y) & :=\frac{H^{2 p-1-n}(Y, \mathbb{C})}{F^{p} H^{2 p-1-n}(Y, \mathbb{C})+H^{2 p-1-n}(Y, \mathbb{Q}(p))} \\
& \simeq \frac{H^{2 p-1}\left(\square_{Y}^{n}, \partial \square_{Y}^{n} ; \mathbb{C}\right)}{F^{p} H^{2 p-1}\left(\square_{Y}^{n}, \partial \square_{Y}^{n} ; \mathbb{C}\right)+H^{2 p-1}\left(\square^{n}, \partial \square_{Y}^{n} ; \mathbb{Q}(p)\right)} \\
& \simeq \frac{H^{2 p-1}\left(\square_{Y}^{n}-|Z|, \partial \square_{Y}^{n}-|Z| ; \mathbb{C}\right)}{F^{p} H^{2 p-1}\left(\square_{Y}^{n}-|Z|, \partial \square_{Y}^{n}-|Z| ; \mathbb{C}\right)+H^{2 p-1}\left(\square^{n}, \partial \square^{n} ; \mathbb{Q}(p)\right)} .
\end{aligned}
$$

Suppose that $Z \in \operatorname{Ker} \partial_{\square} \subset z^{p}(Y, n)^{\text {alt }}$. Since $Z \in z^{p}(Y, n)^{\text {alt }}$, we have $\partial_{i} Z=0$ for each codimension one face $H_{i}$ and so we have a class $\operatorname{cl}(Z) \in H_{|Z|}^{2 p}\left(\square_{Y}^{n}, \partial \square_{Y}^{n} ; \mathbb{Q}(p)\right)$. If $Z$ is homologous to zero i.e. $\iota(\operatorname{cl}(Z))=0$, there is a class $\widetilde{c l(Z)} \in H^{2 p-1}\left(\square_{Y}^{n}-|Z|, \partial \square_{Y}^{n}-\right.$ $|Z| ; \mathbb{Q}(p))$ whose image under the boundary map is $c l(Z)$. We denote by $z_{\text {hom }}^{p}(Y, n)$ the subspace $\operatorname{Ker} \partial_{\square} \cap \operatorname{Ker} \iota$ of $z^{p}(Y, n)^{\text {alt }}$.

Definition 4.2. The map

$$
\Psi^{p, n}: z_{\mathrm{hom}}^{p}(Y, n) \rightarrow J^{p, n}(Y), Z \mapsto \text { the class of } \widetilde{\operatorname{cl}(\mathrm{Z})}
$$

is called the Abel-Jacobi map.

Remark 4.3. One can also define the Abel-Jacobi map in terms of Deligne-Beilinson cohomology. The equality of the two definitions is proved in Theorem 7.11 [5]. 
4.1. An explicit description of the Abel-Jacobi map. We will give a more explicit description of the map $\Psi^{p, n}$. Let $Z$ be an element of $z_{\text {hom }}^{p}(Y, n)$. We replace the complex $A C^{*}\left(\square_{Y}^{n}, \partial \square_{Y}^{n}\right)$ with a smaller complex $A C_{\square}^{*}\left(\square^{n} Y\right)$. In the following, the coefficients of the complex $A C^{*}$ will be $\mathbb{Q}$.

Definition 4.4. Let $A C_{\square}^{*}\left(\square_{Y}^{n}\right)$ be the simple complex associated to the double complex

$$
A C_{\square}^{a, b}\left(\square_{Y}^{n}\right)=A C_{2(m+n-a)-b}\left(\left(\mathbb{P}^{1}\right)_{Y}^{n-a}, D_{Y}\right)^{a l t} \quad(a \geq 0)
$$

where the first differential is $\partial_{\square}$ and the second differential is $\delta$.

Proposition 4.5. Let the map $\sum: A C^{k}\left(\square^{n_{Y}}, \partial \square^{n_{Y}}\right) \rightarrow A C_{\square}^{k}\left(\square^{n_{Y}}\right)$ be the map defined as

$$
\gamma_{\left(\alpha_{0}<\cdots<\alpha_{p}\right)} \mapsto(-1)^{\sum_{j=0}^{p} \alpha_{j}} \gamma
$$

Then the map Alto $\sum$ is a quasi-isomorphism.

Proof. We can argue as in [16] 1.7. That the map Alto $\sum$ is a map of complexes can be checked directly. The cohomology group $H^{k}\left(A C\left(\square_{Y}^{n_{Y}}, \partial \square_{Y}^{n_{Y}}\right)\right)$ can be computed in terms of the spectral sequence

$$
E_{0}^{a b}=\underset{I \subset T, \sharp I=a}{\oplus} A C_{2(m+n-a)-b}\left(\left(\mathbb{P}^{1}\right)_{Y}^{n-a}, D_{Y}\right) .
$$

By the homotopy invariance one sees that

$$
E_{1}^{a b}=\underset{I \subset T, \sharp I=a}{\oplus} H^{b}(Y, \mathbb{Q})
$$

and

$$
E_{2}^{a b}=H^{b}(Y, \mathbb{Q}) \otimes H^{a}\left(\square^{n}, \partial \square^{n} ; \mathbb{Q}\right)= \begin{cases}0 & a \neq n \\ H^{b}(Y, \mathbb{Q}) & a=n .\end{cases}
$$

On the other hand, the cohomology of $A C_{\square}^{*}\left(\square^{n} Y\right)$ is computed by the spectral sequence

$$
\square E_{0}^{a b}=A C_{2(m+n-a)-b}\left(\left(\mathbb{P}^{1}\right)_{Y}^{n-a}, D_{Y}\right)^{\text {alt }} .
$$

We see that $\square E_{1}^{a b}=H^{b}\left(\square^{n-a} Y ; \mathbb{Q}\right)^{\text {alt }}=0$ unless $a=n$. We see the map Alt $\circ \sum$ induces an isomorphism on the $E_{2}$-terms of the spectral sequences.

Proposition 4.6. Let $Z \in z^{p}(Y, n)^{\text {alt }}$.

(1) We have

$$
H^{j}\left(\operatorname{Cone}\left(A C_{\square}^{*}\left(\square_{Y}^{n}\right) \rightarrow A C_{\square}^{*}\left(\square_{Y}^{n}-|Z|\right)\right)\right)=0
$$

for $j<2 p$, and

$$
\begin{aligned}
& H^{2 p}\left(\operatorname{Cone}\left(A C_{\square}^{*}\left(\square_{Y}^{n}\right) \rightarrow A C_{\square}^{*}\left(\square_{Y}^{n}-|Z|\right)\right)\right) \\
\simeq \quad & H_{|Z|}^{2 p}\left(\square_{Y}^{n}, \partial \square_{Y}^{n} ; \mathbb{Q}\right)^{\text {alt }}
\end{aligned}
$$

$(2)$

$$
H^{2 p-1}\left(A C_{\square}^{*}\left(\square_{Y}^{n}-|Z|\right)\right) \simeq H^{2 p-1}\left(\square_{Y}^{n}-|Z|, \partial \square_{Y}^{n}-|Z| ; \mathbb{Q}\right)^{\text {alt }} .
$$


Proof. (1). There is a spectral sequence

$$
\begin{aligned}
& E_{0}^{a b}=A C_{2(m+n-a)-b}\left(\left(\mathbb{P}^{1}\right)_{Y}^{n-a}, D ; \mathbb{Q}\right) \oplus A C_{2(m+n-a)-b+1}\left(\left(\mathbb{P}^{1}\right)_{Y}^{n-a}, D \cup|Z| ; \mathbb{Q}\right) \\
& \Rightarrow H^{a+b}\left(\operatorname{Cone}\left(A C_{\square}^{*}\left(\square_{Y}^{n}\right) \rightarrow A C_{\square}^{*}\left(\square_{Y}^{n}-|Z|\right)\right)\right) .
\end{aligned}
$$

We have

$$
E_{1}^{a b}=H_{|Z| \cap \square^{n-a} Y}^{b}\left(\square^{n-a} Y ; \mathbb{Q}\right)^{\text {alt }} .
$$

By purity, $E_{1}^{a b}=0$ for $b<2 p$. It follows that

$$
\begin{aligned}
& H^{2 p}\left(\operatorname{Cone}\left(A C_{\square}^{*}\left(\square_{Y}^{n}\right) \rightarrow A C_{\square}^{*}\left(\square_{Y}^{n}-|Z|\right)\right)\right) \\
\simeq \quad & E_{2}^{02 p}=\operatorname{Ker}\left(\partial_{\square}: H_{|Z|}^{2 p}\left(\square_{Y}^{n} ; \mathbb{Q}\right)^{\text {alt }} \rightarrow H_{|Z| \cap \square^{n-1} Y}^{2 p}\left(\square^{n-1} Y ; \mathbb{Q}\right)^{\text {alt }}\right) .
\end{aligned}
$$

The assertion (1) follows this and Lemma 4.1 (2).

(2). We have exact sequences

$$
\begin{gathered}
(\mathrm{A}): \quad 0 \rightarrow H^{2 p-1}\left(\square_{Y}^{n}, \partial \square_{Y}^{n}\right)^{\text {alt }} \rightarrow H^{2 p-1}\left(\square_{Y}^{n}-|Z|, \partial \square_{Y}^{n}-|Z|\right)^{\text {alt }} \\
\rightarrow H_{|Z|}^{2 p}\left(\square_{Y}^{n}, \partial \square_{Y}^{n}\right)^{\text {alt }} \rightarrow H^{2 p}\left(\square_{Y}^{n}, \partial \square_{Y}^{n}\right)^{\text {alt }} \\
\begin{aligned}
(\mathrm{B}): \quad & 0 \rightarrow H^{2 p-1}\left(A C_{\square}^{*}\left(\square_{Y}^{n}\right)\right) \rightarrow H^{2 p-1}\left(A C_{\square}^{*}\left(\square_{Y}^{n}-|Z|\right)\right) \\
& \rightarrow H^{2 p}\left(C o n e\left(A C_{\square}^{*}\left(\square_{Y}^{n}\right) \rightarrow A C_{\square}^{*}\left(\square_{Y}^{n}-|Z|\right)\right)\right) \\
& \rightarrow H^{2 p}\left(A C_{\square}^{*}\left(\square_{Y}^{n}\right)\right) .
\end{aligned}
\end{gathered}
$$

We have a map of complexes $(\mathrm{A}) \rightarrow(\mathrm{B})$ induced by the map Alt $\circ \Sigma$. The assertion (2) follows from the five lemma.

As is defined in [2], the cohomology $\operatorname{class} c l(Z) \in H_{|Z|}^{2 p}\left(\square^{n} Y, \partial \square_{Y}^{n} ; \mathbb{Q}\right)$ is represented by the cocycle

$$
(Z, 0) \in \operatorname{Cone}^{2 p}\left(A C_{\square}^{*}\left(\square^{n} Y\right) \rightarrow A C_{\square}^{*}\left(\square^{n} Y-|Z|\right)\right) .
$$

Since $Z \in z_{\text {hom }}^{p}(Y, n)$, there is an element $\Gamma \in A C_{\square}^{2 p-1}\left(\square^{n}{ }^{n}\right)$ such that $d \Gamma=Z$. By definition $\Psi^{p, n}(Z)$ is the class of $\Gamma$ in $J^{p, n}(Y)$. We will give a description of $\Psi^{p, n}(Z)$ in terms of currents.

We have

$$
\begin{aligned}
J^{p, n}(Y) & =\frac{H^{2 p-1-n}(Y, \mathbb{C})}{F^{p} H^{2 p-1-n}(Y, \mathbb{C})+H^{2 p-1-n}(Y, \mathbb{Q}(p))} \\
& \simeq \frac{H^{2 p-1}\left(\square_{Y}^{n}, \partial \square_{Y}^{n} ; \mathbb{C}\right)^{\text {alt }}}{\left(F^{p} H^{2 p-1}\left(\square_{Y}^{n}, \partial \square_{Y}^{n} ; \mathbb{C}\right)+H^{2 p-1}\left(\square^{n}{ }_{Y}, \partial \square^{n} ; \mathbb{Q}(p)\right)\right)^{\text {alt }}} \\
& \simeq \frac{H^{2 p-1}\left(\left(\mathbb{P}^{1}\right)_{Y}^{n}, \partial\left(\mathbb{P}^{1}\right)_{Y}^{n} ; \mathbb{C}\right)^{\text {alt }}}{\left(F^{p} H^{2 p-1}\left(\left(\mathbb{P}^{1}\right)_{Y}^{n}, \partial\left(\mathbb{P}^{1}\right)_{Y}^{n} ; \mathbb{C}\right)+H^{2 p-1}\left(\mathbb{P}_{Y}^{1}, \partial\left(\mathbb{P}^{1}\right)_{Y}^{n} ; \mathbb{Q}(p)\right)\right)^{\text {alt }}} \\
& \simeq \frac{\left(F^{m+n-p+1} H^{2(m+n-p)+1}\left(\left(\left(\mathbb{P}^{1}\right)^{n}-\partial\left(\mathbb{P}^{1}\right)^{n}\right)_{Y}, \mathbb{C}\right)^{\text {alt }}\right)^{V}}{H_{2(m+n-p)+1}\left(\left(\left(\mathbb{P}^{1}\right)^{n}-\partial\left(\mathbb{P}^{1}\right)^{n}\right)_{Y}, \mathbb{Q}(p)\right)^{\text {alt }}} .
\end{aligned}
$$

We denote by $\omega_{n}$ the meromorphic form $\frac{1}{(2 \pi i)^{n}} \frac{d z_{1}}{z_{1}} \wedge \cdots \wedge \frac{d z_{n}}{z_{n}}$ on $\square^{n}$. The map

$$
A^{*}(Y) \rightarrow A^{*}\left(\left(\left(\mathbb{P}^{1}\right)^{n}-\partial\left(\mathbb{P}^{1}\right)^{n}\right)_{Y}\right)^{\text {alt }}[n], \varphi \mapsto p_{1}^{*} \varphi \wedge \omega_{n}
$$

is a canonical quasi-isomorphism. Here the map $p_{1}:\left(\left(\mathbb{P}^{1}\right)^{n}-\partial\left(\mathbb{P}^{1}\right)^{n}\right)_{Y} \rightarrow Y$ is the projection. 
Proposition 4.7. Let $\varphi$ be a smooth $p$-form on $Y$ and $\gamma$ be an element of $A C_{p+1+n}\left(\square_{Y}^{n}\right)^{\text {alt }}$. Then under the notation of Notation 3.1, we have the equality

$$
(-1)^{p}\left(\partial_{\square} \gamma, \varphi \wedge \omega_{n-1}\right)=\left(\delta \gamma, p_{1}^{*} \varphi \wedge \omega_{n}\right)-\left(\gamma, p_{1}^{*} d \varphi \wedge \omega_{n}\right)
$$

Proof. The proof is essentially the same as that of Theorem 4.3 [8], with the necessary modification similar to the case of the proof of Proposition 3.2.

Corollary 4.8. Let $\epsilon_{3}$ be a function on $A C_{\square}^{*}(Y)$ which takes the value $\frac{j(j+1)}{2}+a$ on $A C_{j}\left(\square_{Y}^{n-a}\right)^{\text {alt }}$. Then the pairing

$$
(\bullet, \bullet)_{\square}, A C_{\square}^{*}\left(\square_{Y}^{n}\right) \otimes A^{*}(Y) \rightarrow \mathbb{C}[-2 m-n]
$$

which sends $\gamma \otimes \varphi$ with $\gamma \in A C_{j+n-a}\left(\left(\mathbb{P}^{1}\right)_{Y}^{n-a}, D_{Y}\right)^{\text {alt }}$ and $\varphi \in A^{j}(Y)$ to $(-1)^{\epsilon_{3}(\gamma)} \int_{\gamma} p_{1}^{*} \varphi \wedge$ $\omega_{n-a}$ is a map of complexes.

Corollary 4.9. Let $Z \in \operatorname{Ker} \partial_{\square} \subset z^{p}(Y, n)_{h o m}^{\text {alt }}$ and $\Gamma \in A C_{\square}^{2 p-1}\left(\square_{Y}^{n}\right)$ be an element such that $d \Gamma=Z$.

(1) The map $\varphi \mapsto(\Gamma, \varphi) \square$ gives a well defined element of $\left(F^{m-p+1} H^{2(m-p)+n+1}(Y, \mathbb{C})\right)^{\vee}$.

(2) Modulo $H_{2(m-p)+n+1}(Y, \mathbb{Q}(p))$ this element of $(1)$ is independent of the choice of $\Gamma$. Hence this map defines a well defined element of $J^{p, n}(Y)$, which is equal to $\Psi_{p, n}(Z)$.

Proof. (1). If a closed form $\varphi \in F^{m-p+1} A^{2(m-p)+n+1}(Y)$ is exact, $\varphi=d \psi$ for an element $\psi \in F^{m-p+1} A^{2(m-p)+n}(Y)$ by Hodge theory. We have

$$
(\varphi, \Gamma)_{\square}= \pm(\psi, d \Gamma)_{\square}= \pm(\psi, Z)_{\square}=0
$$

for reason of type.

(2). If $d \Gamma^{\prime}=Z$, then $\Gamma-\Gamma^{\prime}$ defines an element of

$$
\begin{aligned}
& H^{2 p-1}\left(\square_{Y}^{n}, \partial \square_{Y}^{n} ; \mathbb{Q}(p)\right)^{\text {alt }} \\
\simeq & H^{2 p-1}\left(\left(\mathbb{P}^{1}\right)_{Y}^{n}, \partial\left(\mathbb{P}^{1}\right)_{Y}^{n} ; \mathbb{Q}(p)\right)^{\text {alt }} \\
\simeq & H_{2(m+n-p)+1}\left(\left(\left(\mathbb{P}^{1}\right)^{n}-\partial\left(\mathbb{P}^{1}\right)^{n}\right)_{Y}, \mathbb{Q}(p)\right)^{\text {alt }} \\
\simeq & H_{2(m-p)+n+1}(Y, \mathbb{Q}(p)) .
\end{aligned}
$$

We will describe how the chain $\Gamma$ looks. Since $H^{j}\left(\square_{Y}^{n}, \mathbb{Q}\right)^{\text {alt }}=0$ for $n>0$, there is an element $\gamma_{n} \in A C_{2(m+n-p)+1}\left(\square_{Y}^{n}\right)$ with $\delta \gamma_{n}=Z$. Since $\delta \partial_{\square} \gamma_{n}=\partial_{\square} \delta \gamma_{n}=\partial_{\square} Z=0$, if $n-1>0$ there is an element $\gamma_{n-1} \in A C_{2(m+n-p)-1}\left(\square_{Y}^{n-1}\right)$ with $\delta \gamma_{n-1}=\partial_{\square} \gamma_{n}$. One inductively finds chains $\gamma_{j} \in A C_{2(m-p)+n+j+1}\left(\square_{Y}^{j}\right), j=1, \cdots, n$ such that $\partial_{\square} \gamma_{j+1}=\delta \gamma_{j}$. The chain $\partial \gamma_{1} \in C_{2(m-p)+n}(Y)$ defines the homology class of $Z$ in $H_{2(m-p)+n}(Y, \mathbb{Q})$ up to sign. Since $Z \in z^{p}(Y, n)_{h o m}^{\text {alt }}$, there is a chain $\gamma_{0} \in C_{2(m-p)+n+1}(Y)$ such that $\partial_{\square} \gamma_{1}=\delta \gamma_{0}$. Then the chain $\Gamma$ equals the sum $\sum_{j=0}^{n}(-1)^{\frac{j(j-1)}{2}} \gamma_{n-j}$. Hence we have the following.

\section{Corollary 4.10.}

$$
\Psi^{p, n}(Z)(\varphi)=\sum_{j=0}^{n}(-1)^{\epsilon_{3}\left(\gamma_{n-j}\right)+\frac{j(j-1)}{2}} \int_{\gamma_{n-j}} p_{1}^{*} \varphi \wedge \omega_{n-j}
$$


Next we consider the Abel-Jacobi map for open varieties, and of the relative higher Chow cycles. Let $Y$ be a smooth projective variety, and suppose that $\mathbf{D}=D_{0} \cup D_{1} \cup \cdots \cup D_{s}$ and $V=V_{0} \cup \cdots \cup V_{t}$ are strict normal crossing divisors such that $\mathbf{D} \cup V$ is also a strict normal crossing divisor. We denote $U=Y-\mathbf{D}, S=\{0, \cdots, s\}$ and $T=\{0, \cdots, t\}$. The subvariety of $Y$ of the form $V_{I}:=\cap_{i \in I} V_{i}$ for a subset $I$ of $T$, is called a $V$-face. A face of $\square^{l}$ is called a cubical face. The variety $\square^{l}$ itself is a cubical face. For a subset $I$ of $T$ and a subset $J$ of $S$, a face of $\left(D_{J} \cap V_{I}\right) \times \square^{l}$ is the product of a $V$-face and a cubical face. Let $z^{r}\left(D_{J} \cap V_{I}, l\right)_{V}^{\text {alt }}$ be the subspace of $z^{r}\left(D_{J} \cap V_{I}, l\right)^{\text {alt }}$ which consists of the cycles $z$ which meet all the faces of $\left(D_{J} \cap V_{I}\right) \times \square^{l}$ properly. By the moving Lemma ([1], [13]), the complex $z^{r}\left(D_{J} \cap V_{I}, *\right)_{V}^{\text {alt }}$ with the differential $\partial_{\square}$ is quasi-isomorphic to $z^{r}\left(D_{J} \cap V_{I}, *\right)^{\text {alt }}$. Let $z^{r}(U, V, *)$ be the complex defined by the equality

$$
z^{r}(U, V, n)=\underset{\substack{I \subset T, J \subset S \\ \sharp J-\sharp I+l=n}}{\bigoplus} z^{r-\sharp J}\left(D_{J} \cap V_{I}, l\right)_{V}^{\text {alt }}
$$

where the differential is defined by

$$
d=\partial_{D}+(-1)^{\sharp J} \partial_{V}+(-1)^{\sharp J+\sharp I} \partial_{\square}
$$

on $z^{r-\sharp J}\left(D_{J} \cap V_{I}, m\right)_{V}$. Then the higher Chow group of $U$ relative to $V$ is defined by the equality

$$
C H^{r}(U, V ; n)=H_{n}\left(z^{r}(U, V, *)\right) \text {. }
$$

Accordingly we consider the following complex of admissible chains.

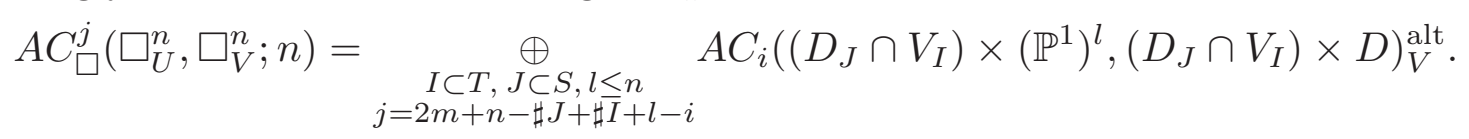

Here $A C_{i}\left(\left(D_{J} \cap V_{I}\right) \times\left(\mathbb{P}^{1}\right)^{l},\left(D_{J} \cap V_{I}\right) \times D\right)_{V}^{\text {alt }}$ is the subspace of $A C_{i}\left(\left(D_{J} \cap V_{I}\right) \times\left(\mathbb{P}^{1}\right)^{l},\left(D_{J} \cap\right.\right.$ $\left.\left.V_{I}\right) \times D\right)^{\text {alt }}$ which consists of the chains $\gamma$ such that $|\gamma|$ and $|\delta \gamma|$ meet all the faces of $\left(D_{J} \cap V_{I}\right) \times \square^{l}$ properly. The differential is defined by

$$
d=\partial_{D}+(-1)^{\sharp J} \partial_{V}+(-1)^{\sharp J+\sharp I} \partial_{\square}+(-1)^{\sharp J+\sharp I+l} \delta .
$$

Then we have

$$
H^{j}\left(A C_{\square}^{*}\left(\square_{U}^{n}, \square_{V}^{n}\right)\right)=H^{j}\left(\square_{U}^{n},\left(\square_{V}^{n}\right) \cup\left(\partial \square_{U}^{n}\right) ; \mathbb{Q}^{\text {alt }}\right) .
$$

In this case we also have a description of the Abel-Jacobi map in terms of the admissible chains. Probably it is more adequate to give an example than writing down the general formula. In the next subsection we will describe the case of polylog cycles rather explicitly.

4.2. The case of polylogarithms. We will see that the Hodge realization of polylog cycle constructed in [3] can be described in terms of the Abel-Jacobi map. Fix an integer $p \geq 1$, and for $1 \leq k \leq p$ let $Y_{k}=\left(\mathbb{P}^{1}\right)^{k-1}$ with the affine coordinates $\left(x_{1}, \cdots, x_{k-1}\right)$. Let $a \in \mathbb{C}-\{0,1\}$. For $1 \leq j \leq k-1$ and $u \in\left\{a, a^{-1}\right\}$ let $D_{j, u}$ be the divisor on $Y_{k}$ defined by $x_{j}=u$. Then the closed subset

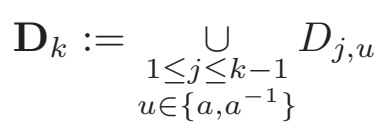

is a normal crossing divisor of $Y_{k}$. We write the set of indices of the irreducible components of $\mathbf{D}_{p}$

$$
\left\{(j, u) \mid 1 \leq j \leq p-1, u \in\left\{a, a^{-1}\right\}\right\}
$$


by $S$, and define an ordering on $S$ by

$$
(1, a)<\left(1, a^{-1}\right)<\left(2, a^{-1}\right)<(2, a)<(3, a)<\left(3, a^{-1}\right)<\left(4, a^{-1}\right) \cdots
$$

where the numbering starts from zero. We denote $Y_{k}-\mathbf{D}_{k}$ by $U_{k}$. We have

$$
C H^{p}\left(U_{p}, p\right)=H_{p}(Z(*))
$$

where

$$
Z(n)=\bigoplus_{\substack{J \subset S, l \geq 0 \\ \sharp J+l=n}} z^{p-\sharp J}\left(D_{J}, l\right)^{\text {alt }} .
$$

with the differential $d=\partial_{D}+(-1)^{\sharp J} \partial_{\square}$ on $z^{k}\left(D_{J}, l\right)$. The group $G_{k-1}$ acts on $Y_{k}$ in a similar way as $G_{l}$ acts on $\square^{l}$, so there is an action of the product $G_{k-1} \times G_{l}$ on $Y_{k} \times \square^{l}$. The character sign naturally extends to the product character $\operatorname{sign}_{\times}$on $G_{k-1} \times G_{l}$, and let $\mathrm{Alt}_{\times}$be the associated projector. For a $\mathbb{Q}\left[\left(G_{k-1} \times G_{l}\right)\right]$-module $M$, we denote by $M^{\text {alt }} \times$ the module $\operatorname{Alt}_{\times}(M)$. Let $\mathcal{Z}(*)$ be the complex defined by the equality

$$
z(n)=\bigoplus_{0 \leq c \leq n} z^{p-c}\left(Y_{p-c}, n-c\right)^{\text {alt } \times} .
$$

The differential of $z(*)$ is defined as follows. For $u \in\left\{a, a^{-1}\right\}$ and $j \geq 1$, let $\iota_{u}^{j}: Y_{k} \rightarrow Y_{k+1}$ be the inclusion defined by $\left(x_{1}, \cdots, x_{k-1}\right) \mapsto\left(x_{1}, \cdots, x_{j-1}, u, x_{j+1}, \cdots, x_{k-1}\right)$. Let

$$
\partial_{Y}: z^{\bullet}\left(Y_{k}, l\right) \rightarrow z^{\bullet+1}\left(Y_{k+1}, l\right)
$$

be the map $\sum_{j=1}^{k-1}(-1)^{j-1}\left(\left(\iota_{a}^{j}\right)_{*}-\left(\iota_{a^{-1}}^{j}\right)_{*}\right)$. The map $\partial_{Y}$ is a differential i.e. $\partial_{Y}^{2}=0$, and it induces a map on the alternating part: we have $\partial_{Y}\left(z^{\bullet}\left(Y_{k}, l\right)^{\text {alt } \times}\right) \subset z^{\bullet+1}\left(Y_{k+1}, l\right)^{\text {alt }} \times$. Let

$$
s: Z(*) \rightarrow Z(*)
$$

be the homomorphism defined as follows. For a subset $J=\left\{\beta_{0}<\cdots<\beta_{c-1}\right\}$ of $S$, let $D_{J}$ be the corresponding face of $Y_{p}$. We identify $D_{J}$ with $Y_{p-c}=\left(\mathbb{P}^{1}\right)^{p-1-c}$ in the natural way. Then on $z^{p-c}\left(Y_{p-c}, l\right)^{\text {alt }}$, we define

$$
s(z)=\sum_{J=\left\{\beta_{0}<\cdots<\beta_{c-1}\right\}}\left((-1)^{\sum_{j=1}^{c-1} \beta_{j}} z \in z^{p-c}\left(D_{J}, n-c\right)^{\text {alt }}\right) .
$$

One sees that the map $s$ is a homomorphism of complexes.

Let $\rho_{k}(a)$ be an element of $z^{k}\left(Y_{k}, k\right)^{\text {alt }} \times$ given parametrically

$$
(-1)^{p-k}\left\{\left(x_{1}, \cdots, x_{k-1}, 1-x_{1}, 1-\frac{x_{2}}{x_{1}}, \cdots, 1-\frac{x_{k-1}}{x_{k-2}}, 1-\frac{a}{x_{k-1}}\right)\right\}^{\text {alt }} \times
$$

we have the equality $\partial_{\square} \rho_{p-c}(a)=\partial_{Y} \rho_{p-c-1}(a)$ for $0 \leq c \leq p-2$. Let $R_{p}(a) \in \mathcal{Z}(p)$ be the element $\sum_{c=0}^{p-1}(-1)^{\frac{c(c+1)}{2}} \rho_{p-c}(a)$. Then we have $d R_{p}(a)=0$.

For $k \geq 2$ or $i>0$ we have $H^{j}\left(Y_{k} \times \square^{i}, \mathbb{Q}\right)^{\text {alt }} \times=0$. So we can find chains

$$
\eta_{p-c}(i) \in A C_{p-c+i}\left(Y_{p-c} \times\left(\mathbb{P}^{1}\right)^{i+1}, Y_{p-c} \times D\right)^{\text {alt } \times}
$$

for $0 \leq c \leq p-1$ and $0 \leq i \leq p-c-1$ such that

$$
\begin{aligned}
\delta \eta_{p-c}(p-c-1) & =\rho_{p-c}(a) \\
\delta \eta_{p-c}(i) & =\partial_{Y} \eta_{p-c-1}(i)+(-1)^{p-c+i+1} \partial_{\square} \eta_{p-c}(i+1), 0 \leq i \leq p-c-2 .
\end{aligned}
$$


Explicitly,

$$
\begin{aligned}
& \eta_{p-c}(i) \\
= & \left\{\left(x_{1}, \cdots, x_{i}, t_{i+1}, \cdots, t_{p-c-1}, 1-x_{1}, \cdots, 1-\frac{x_{i}}{x_{i-1}}, 1-\frac{t_{i}}{x_{i}}\right) \mid\right. \\
& t_{p-c-1} \in[0, a], \cdots, t_{k-2} \in\left[0, t_{k-1}\right], \cdots, t_{i} \in\left[0, t_{i+1}\right], \\
& \left.x_{1}, \cdots, x_{i} \in \mathbb{P}^{1}\right\}^{\text {alt }} .
\end{aligned}
$$

with suitable orientation. A complex computing the cohomology group $H^{\bullet}\left(\square_{U_{p}}^{p}, \partial \square_{U_{p}}^{p} ; \mathbb{Q}\right)$ is given by

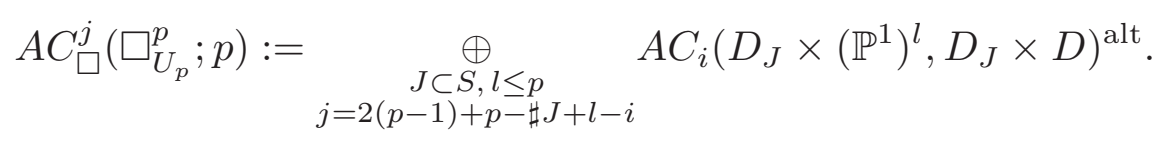

where the differential is given by $\partial_{D}+(-1)^{\sharp J} \partial_{\square}+(-1)^{\sharp J+l} \delta$ on $A C_{i}\left(D_{J} \times\left(\mathbb{P}^{1}\right)^{l}, D_{J} \times D\right)^{\text {alt }}$. Let $\mathcal{A} C^{\bullet}$ be the complex defined by

$$
\mathcal{A} C^{j}=\bigoplus_{\substack{0 \leq c \leq p-1, l \leq p \\ j=2(p-1)+p-c+l-i}} A C_{i}\left(Y_{p-c} \times\left(\mathbb{P}^{1}\right)^{l}, Y_{p-c} \times D\right)^{\text {alt } \times}
$$

with the differential given by $\partial_{Y}+(-1)^{c} \partial_{\square}+(-1)^{c+l} \delta$ on $A C_{i}\left(Y_{p-c} \times\left(\mathbb{P}^{1}\right)^{l}, Y_{p-c} \times D\right)^{\text {alt }} \times$. The map $s: \mathcal{A} C^{\bullet} \rightarrow A C^{\bullet}\left(\square_{U_{p}}^{p} ; p\right)$ defined in (4.2.3) is a homomorphism of complexes. The element $R_{p}(a)$ can be regarded as a cocycle of $\mathcal{A} C^{2 p}$. Let $y_{p}(a)$ be the element of $\mathcal{A} C^{2 p-1}$ defined as the sum

$$
\sum_{0 \leq c \leq p-1,0 \leq i \leq p-c-1}(-1)^{\epsilon(c, i)} \eta_{p-c}(i)
$$

where $\epsilon(c, i):=1+i(c+1+p)+\frac{c(c-1)}{2}$. Then we have

$$
d y_{p}(a)=R_{p}(a)
$$

Let $V$ be the divisor

$$
\bigcup_{i=1}^{p-1}\left(\left\{x_{i}=0\right\} \cup\left\{x_{i}=\infty\right\}\right)
$$

of $Y_{p}$. For each irreducible component $V_{i}$ of $V$, the pull-back of $s\left(\rho_{p-c}(a)\right)$ and of $s\left(\eta_{p-c}(i)\right)$ to $V_{i}$ is zero for each $0 \leq c \leq p-1$ and $0 \leq i \leq p-c-1$. Hence $s\left(R_{p}(a)\right)$ defines an element of the relative higher Chow group $C H^{p}\left(U_{p}, V ; p\right)$ and we have

$$
d s\left(y_{p}(a)\right)=s\left(R_{p}(a)\right)
$$

in $A C_{\square}^{2 p}\left(\square_{U_{p}}^{p}, \square_{V}^{p} ; p\right)$. We have

$$
H^{2 p-1}\left(\square_{U_{p}}^{p},\left(\square_{V}^{p}\right) \cup\left(\partial \square_{U_{p}}^{p}\right) ; \mathbb{Q}\right)^{\text {alt }} \simeq H^{p-1}\left(U_{p}, V ; \mathbb{Q}\right) .
$$

The dual space of $H^{p-1}\left(U_{p}, V ; \mathbb{Q}\right) \otimes \mathbb{C}$ is $H^{p-1}\left(\left(\mathbb{P}^{1}\right)^{p-1}-V, \mathbf{D}_{p} ; \mathbb{C}\right)$. The form $\omega_{p-\sharp J-1}$ on $D_{J}$ defines an element of $H^{p-1}\left(\left(\mathbb{P}^{1}\right)^{p-1}-V ; \mathbf{D}_{p} ; \mathbb{C}\right)$. We have

$$
\left(s\left(y_{p}(a)\right),\left(\omega_{p-\sharp J-1} \text { on } D_{J}\right)\right)=\sum_{i=0}^{p-\sharp J-1}(-1)^{\epsilon(\sharp J, i)} \int_{\eta_{p-\sharp J}(i)} \omega_{p-\sharp J-1} \wedge \omega_{i+1} .
$$


When $i>0$, the integral $\int_{\eta_{p-\sharp J}(i)} \omega_{p-\sharp J-1} \wedge \omega_{i+1}=0$ for reason of type, and

$$
\begin{aligned}
& \int_{\eta_{p-\sharp J}(0)} \omega_{p-\sharp J-1} \wedge \omega_{1} \\
= & \frac{1}{(2 \pi i)^{p-\sharp J}} \int_{0}^{a} \frac{d t_{p-\sharp J-1}}{t_{p-\sharp J-1}} \cdots \int_{0}^{t_{2}} \frac{d t_{1}}{t_{1}} \int_{0}^{t_{1}} \frac{-d t_{0}}{1-t_{0}} \\
= & \frac{1}{(2 \pi i)^{p-\sharp J}} L i_{p-\sharp J}(a) .
\end{aligned}
$$

\section{REFERENCES}

[1] Bloch,S., The moving lemma for higher Chow groups, J. Algebraic Geom. 3, (1994), 537-568.

[2] Bloch, S., Algebraic cycles and the Beilinson conjectures, Contemporary Mathematics 58 (1) (1986), 65-79.

[3] Bloch, S., Kriz, I., Mixed Tate Motives, Ann. Math. 140, 557-605.

[4] Bochnak, J., Coste, M., Roy, M., Real Algebraic Geometry, Springer, 1998

[5] Esnault, H., Vieweg, E., Deligne-Beilinson cohomology, in Beilinson's Conjectures on Special Values of $L$-functions.

[6] Griffiths, A. P., On the periods of certain rational integrals: I, Ann. Math., 90, 460-495, (1969).

[7] Hanamura, M., Kimura, K., Terasoma, T., Integrals of logarithmic forms on semi-algebraic sets and a generalized Cauchy formula, Part I: convergence theorems. arXiv:1509.06950.

[8] Hanamura, M., Kimura, K., Terasoma, T., Integrals of logarithmic forms on semi-algebraic sets and a generalized Cauchy formula, Part II: generalized Cauchy formula (detailed version). arXiv: 1604.03216.

[9] Hatcher, A., Algebraic Topology, Cambridge Univ. Press, 2002.

[10] Kerr, M., Lewis, J., Muller-Stach, S., The Abel-Jacobi map for, higher Chow groups, Compositio Math. 142, (2006), 374-396.

[11] Kerr, M., Lewis, J., The Abel-Jacobi map for higher Chow groups, II, Invent. Math. 170 (2007), no. 2, $355-420$.

[12] King, J., Log complexes of currents and functorial properties of the Abel-Jacobi map, Duke Math. J. 50 (1983), no. 1, 1-53.

[13] Levine, M., Bloch's higher Chow groups revisited, K-theory (Strasbourg, 1992). Astérisque, 226, 235320, Soc. Math. de France, Paris (1994).

[14] Munkres, J., Elements of Algebraic Topology, Addison-Wesley, 1984.

[15] Rourke, C. P., Sanderson, B. J., Introduction to piecewise-linear topology, Ergebnisse der Mathematik und ihrer Grenzgebiete, Band 69, Springer-Verlag, New York-Heidelberg, (1972).

[16] Scholl, A., Extensions of motives, higher Chow groups and special values of L-functions, Séminaire de Théorie des Nombres, Paris, 1991-92, 279-292.

[17] Swan, R.G., The Theory of Sheaves, Chicago Lectures in mathematics, The University of Chicago Press, 1964.

[18] Zeeman, C., Seminar on Combinatorial Topology, Lecture notes, IHES, 1963 